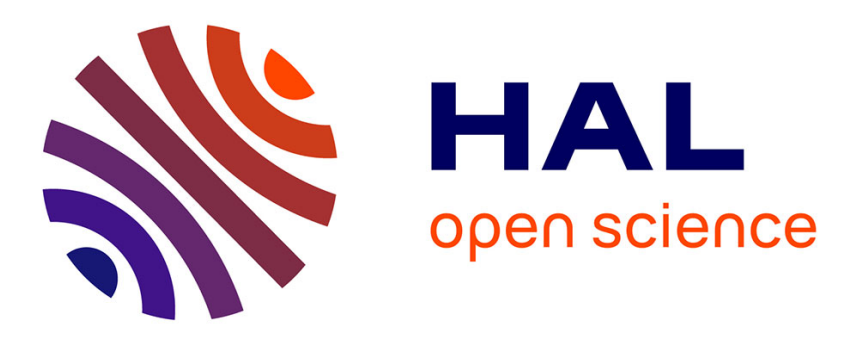

\title{
Mathematical analysis of conductive and superconductive transmission lines
}

Anne-Sophie Bonnet-Ben Dhia, Karim Ramdani

\section{To cite this version:}

Anne-Sophie Bonnet-Ben Dhia, Karim Ramdani. Mathematical analysis of conductive and superconductive transmission lines. SIAM Journal on Applied Mathematics, 2000, 60 (6), pp.2087-2113. $10.1137 /$ S0036139999352420 . hal-01009815

\section{HAL Id: hal-01009815 \\ https://hal-ensta-paris.archives-ouvertes.fr/hal-01009815}

Submitted on 14 Feb 2017

HAL is a multi-disciplinary open access archive for the deposit and dissemination of scientific research documents, whether they are published or not. The documents may come from teaching and research institutions in France or abroad, or from public or private research centers.
L'archive ouverte pluridisciplinaire HAL, est destinée au dépôt et à la diffusion de documents scientifiques de niveau recherche, publiés ou non, émanant des établissements d'enseignement et de recherche français ou étrangers, des laboratoires publics ou privés. 


\title{
MATHEMATICAL ANALYSIS OF CONDUCTING AND SUPERCONDUCTING TRANSMISSION LINES*
}

\author{
ANNE-SOPHIE BONNET-BEN DHIA ${ }^{\dagger}$ AND KARIM RAMDANI ${ }^{\dagger}$
}

\begin{abstract}
This paper is concerned with a mathematical study of guided propagation in the microstrip transmission lines used in microelectronics.

In the first part, the case of a zero-thickness perfectly conducting strip is considered. Using a regularized formulation of Maxwell's equations, it is shown that finding guided modes amounts to the spectral analysis of a noncompact family of self-adjoint operators. The existence of guided modes is then proved thanks to the min-max principle.

In the second part, we deal with the case of a zero-thickness superconducting strip. An asymptotic model derived from London's equation is studied and the existence of guided modes is deduced from the case of the perfectly conducting strip.
\end{abstract}

Key words. superconducting transmission lines, waveguides, spectral analysis, Maxwell's equations

AMS subject classifications. 35P, 47A70, 47B25, 78A50

PII. S0036139999352420

1. Introduction. The wide use of planar transmission lines in microwave integrated circuits has induced the development of various methods to compute their propagation characteristics (cf. [6]). The most common structure is the so-called microstrip line which consists of a thin conducting strip placed on a dielectric substrate located on a conducting ground plane. Electromagnetically guided modes are confined between the ground plane and the strip. Recently, new lines have been realized using superconducting materials instead of conducting ones and providing very low thermic losses.

We are interested here in the theoretical study of guided modes in conducting and superconducting microstrip lines. Throughout the paper, thermic losses are neglected and the conducting strip is considered to be of zero thickness. Moreover, since the modes we are interested in are confined near the strip, the metallic box surrounding the line is omitted. Therefore, the cross section of the line in an unbounded domain. Consequently, as generally in the open waveguides theory (cf. $[1,2,3,5,9]$ ), the determination of the guided modes amounts to solving a noncompact self-adjoint eigenvalue problem.

The first part of the paper is devoted to the case where the strip is supposed to be perfectly conducting. Using a regularized variational formulation for Maxwell's equations, it is proved that the guided modes are related to the eigenvalues of a selfadjoint operator. Since the waveguide is open, this operator has a nonempty essential spectrum. Its determination, which is not so obvious, constitutes the main topic of this part. Then existence results for guided waves are established thanks to the min-

*Received by the editors February 16, 1999; accepted for publication (in revised form) July 23, 1999; published electronically June 15, 2000. A portion of this paper appeared in the Proceedings of the Fourth International Conference on Mathematical and Numerical Aspects of Wave Propagation, Golden, CO, 1998, John A. DeSanto, ed., SIAM, Philadelphia, pp. 12-21.

http://www.siam.org/journals/siap/60-6/35242.html

†Laboratoire de Simulation et Modélisation des Phénomènes de Propagation (associé au Centre National de la Recherche Scientifique), Ecole Nationale Supérieure de Techniques Avancées: ENSTA /SMP, 32 Boulevard Victor, 75739 Paris cédex 15, France (bonnet@ensta.fr, ramdani@ensta.fr). 
max principle. In particular, the existence of the fundamental mode at low frequency is established.

In the second part, the case of a superconducting strip is considered. Since the strip is very thin in practice, its superconducting properties can be modeled thanks to an asymptotic model based on London's equation. This leads us to an impedance boundary condition on the strip. Using the results obtained in the first part, existence results for the guided modes are derived. We conclude the paper by proving that the case of the perfectly conducting strip is the limit, as the impedance parameter goes to infinity, of the case of the superconducting strip.

\section{The perfectly conducting microstrip line.}

2.1. Description of the microstrip line. Let $\left(O, x_{1}, x_{2}, x_{3}\right)$ be an orthonormal system of coordinates. The structure we consider is invariant in the $x_{3}$-direction. It is located in the half-space $x_{2}>0$ and limited in $x_{2}=0$ by a perfectly conducting ground plane. Let

$$
\Omega_{\infty}=\left\{\left(x_{1}, x_{2}\right) ; x_{2}>0\right\}
$$

be the cross section of the line. Throughout this paper, we will assume that the strip is of zero thickness, and its cross section $\Gamma_{S}$ will be considered as a crack. The propagation domain is

$$
\Omega=\Omega_{\infty} \backslash \Gamma_{S} .
$$

Its boundary is $\partial \Omega=\Gamma_{S} \cup \Gamma_{G}$, where $\Gamma_{G}$ denotes the cross section of the ground plane.

The thickness of the dielectric substrate is noted $h$ and the width of the strip $w$ (cf. Figure 1). Furthermore, we suppose that

$$
\Gamma_{S}=\left\{\left(x_{1}, h\right),\left|x_{1}\right|<w / 2\right\} .
$$

The cross section of the dielectric substrate is then $\left.\Omega_{D}=\mathbb{R} \times\right] 0, h[$, and the air's cross section is $\left.\Omega_{A}=\Omega_{\infty} \backslash \overline{\Omega_{D}}=\mathbb{R} \times\right] h,+\infty[$.

In practice, the line is surrounded by a metallic box. Physicists distinguish two kinds of propagating modes in this line: the so-called box modes and the guided modes. The first modes are not well confined near the strip and can interact with the metallic box surrounding the line. On the contrary, the second modes (which are the interesting ones for applications) are perfectly confined and do not "see" the metallic box. The method we propose here to distinguish the guided modes from the others in the mathematical analysis is to consider that the waveguide is an open one and thus to neglect the box. As a consequence, the section of the propagation domain $\Omega$ is supposed to be unbounded. The box modes are no longer guided: they become radiating modes and form the continuous spectrum that will be studied in section 2.4.

2.2. Equations of the guided modes. Guided modes are particular solutions $(\mathbf{E}, \mathbf{H})$ of Maxwell's equations such that

$$
\left\{\begin{array}{l}
\mathbf{E}\left(x_{1}, x_{2}, x_{3}, t\right)=E\left(x_{1}, x_{2}\right) e^{i\left(\omega t-\beta x_{3}\right)} \\
\mathbf{H}\left(x_{1}, x_{2}, x_{3}, t\right)=H\left(x_{1}, x_{2}\right) e^{i\left(\omega t-\beta x_{3}\right)} \\
E, H \in L^{2}(\Omega), \quad \omega, \beta \in \mathbb{R} .
\end{array}\right.
$$

Such solutions describe electromagnetic waves propagating in the $x_{3}$-direction, without attenuation or deformation. $\omega$ is the pulsation, $\beta$ is the propagation constant, 


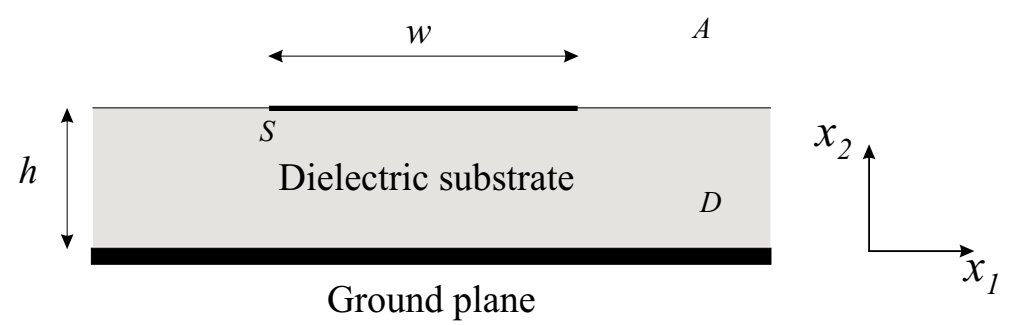

FIG. 1. Cross section of the microstrip transmission line.

and $\omega / \beta$ the phase velocity. The fact that $E$ and $H$ are square integrable means that the transverse energy is finite: this condition is responsible for the confinement of the mode near the strip.

Let $\operatorname{Curl}_{\beta}$ and $\operatorname{Div}_{\beta}$ denote the operators obtained from the classical Curl and Div operators after replacing the derivation, with respect to $x_{3}$, with multiplication by $(-i \beta)$.

Then, from (1) and Maxwell's equations, we deduce the following two-dimensional problem for $\{E, H\}$ :

$$
\begin{cases}\operatorname{Curl}_{\beta} E=-i \omega \mu_{0} H & (\Omega), \\ \operatorname{Cur}_{\beta} H=i \omega \varepsilon \varepsilon_{0} E & (\Omega), \\ \operatorname{Div}_{\beta}(\varepsilon E)=0 & (\Omega), \\ \operatorname{Div}_{\beta} H=0 & (\Omega), \\ E \times n=0 & (\partial \Omega),\end{cases}
$$

where

- $\varepsilon_{0}$ and $\mu_{0}$ are the values of the dielectric permittivity and the magnetic permeability in the vacuum and $\varepsilon$ is the relative dielectric permittivity defined by

$$
\varepsilon=\left\{\begin{array}{ccc}
1 & \text { in } & \Omega_{A} \\
\varepsilon_{D} & \text { in } & \Omega_{D}
\end{array}\right.
$$

where $\varepsilon_{D}$ is a positive constant such that $\varepsilon_{D}>1$.

- $n$ is the unit normal to $\left(\Gamma_{S} \cup \Gamma_{G}\right) \times \mathbb{R}$.

Classically, we deduce from (2) the following problem for the electric field:

$$
\begin{cases}\operatorname{Curl}_{\beta}\left(\operatorname{Curl}_{\beta} E\right)=\omega^{2} \varepsilon E & (\Omega), \\ \operatorname{Div}_{\beta}(\varepsilon E)=0 & (\Omega), \\ E \times n=0 & (\partial \Omega),\end{cases}
$$

where we have supposed, without loss of generality, that $c_{0}^{2}=1 /\left(\varepsilon_{0} \mu_{0}\right)=1$.

2.3. A variational formulation. There are two ways to further expand these ideas. The first consists of introducing a space of fields $E$ such that $\operatorname{Div}_{\beta}(\varepsilon E)=0$ (see, for example, [12]). The numerical counterpart of this choice is the use of edge finite elements. We present here an alternative approach which allows the use of nodal elements $($ see $[8,4])$. In this approach, the divergence-free condition is no longer 
imposed in the variational space, but is taken into account through a penalization term in the variational formulation. Such a formulation is called regularized.

Let us set

$$
\begin{aligned}
& V=\left\{E \in L^{2}(\Omega)^{3} ; \operatorname{Curl}_{\beta} E \in L^{2}(\Omega)^{3}, \operatorname{Div}_{\beta}(\varepsilon E) \in L^{2}(\Omega),\right. \\
&E \times n=0 \text { on } \partial \Omega\} .
\end{aligned}
$$

In fact, $V$ does not depend on $\beta$ and can be defined equivalently by

$$
\begin{aligned}
V=\{ & E \in L^{2}(\Omega)^{3} ; E_{3} \in H^{1}(\Omega), E_{2,1}-E_{1,2} \in L^{2}(\Omega), \\
& \left.\varepsilon E_{1,1}+\left(\varepsilon E_{2}\right)_{, 2} \in L^{2}(\Omega), E \times n=0 \text { on } \partial \Omega\right\} .
\end{aligned}
$$

It is a Hilbert space for the following norm (with the usual notation for the norms in Sobolev spaces):

$$
\|E\|_{V}=\sqrt{\|E\|_{0, \Omega}^{2}+\left\|E_{3}\right\|_{1, \Omega}^{2}+\left\|E_{2,1}-E_{1,2}\right\|_{0, \Omega}^{2}+\left\|\varepsilon E_{1,1}+\left(\varepsilon E_{2}\right)_{, 2}\right\|_{0, \Omega}^{2}},
$$

and the embedding of $V$ in $L_{\text {loc }}^{2}(\Omega)^{3}$ is compact (cf. [16] for the case of homogeneous boundary conditions and [8] for nonhomogeneous ones).

For every $\beta \in \mathbb{R}$, consider the following variational eigenvalue problem:

Find $\omega \in \mathbb{R}$ such that there exists $E \in V, E \neq 0$ satisfying

$$
\int_{\Omega}\left\{\operatorname{Curl}_{\beta} E \cdot \overline{\operatorname{Curl}_{\beta} F}+\operatorname{Div}_{\beta}(\varepsilon E) \overline{\operatorname{Div}_{\beta}(\varepsilon F)}\right\}=\omega^{2} \int_{\Omega} \varepsilon E \cdot \bar{F} \quad \forall F \in V .
$$

Following [4], we can prove the following.

Lemma 2.1. Every solution of (3) satisfies (5). Conversely, if $\omega^{2}<\beta^{2}$, every solution of (5) satisfies (3).

Proof. The fact that every solution of (3) satisfies (5) is a direct consequence of Green's formula. To show the converse result, we first prove that for $\omega^{2}<\beta^{2}$, every solution $E \in V$ of (5) satisfies

$$
\varphi:=\operatorname{Div}_{\beta}(\varepsilon E)=0 \quad(\Omega) .
$$

Indeed, taking as test functions in (5)

$$
F=\operatorname{Grad}_{\beta} \psi,
$$

where $\psi \in H_{0}^{1}(\Omega)$ is such that $\operatorname{Div}_{\beta}\left(\varepsilon \operatorname{Grad}_{\beta} \psi\right) \in L^{2}(\Omega)$, problem (5) reads

$$
\int_{\Omega} \varphi \overline{\operatorname{Div}_{\beta}\left(\varepsilon \operatorname{Grad}_{\beta} \psi\right)}=\omega^{2} \int_{\Omega} \varepsilon E \cdot \overline{\operatorname{Grad}_{\beta} \psi}=-\omega^{2} \int_{\Omega} \varphi \bar{\psi} .
$$

If we define the self-adjoint operator $T$ of $L^{2}(\Omega)$ by

$$
\left\{\begin{array}{l}
D(T)=\left\{\psi \in H_{0}^{1}(\Omega) ; \operatorname{Div}_{\beta}\left(\varepsilon \operatorname{Grad}_{\beta} \psi\right) \in L^{2}(\Omega)\right\}, \\
T \psi=-\operatorname{Div}_{\beta}\left(\varepsilon \operatorname{Grad}_{\beta} \psi\right)-\omega^{2} \psi \quad \forall \psi \in D(T),
\end{array}\right.
$$

then (6) shows that $\varphi \in R(T)^{\perp}$, where $R(T)$ denotes the range of $T$. Thus, $\varphi \in$ $\operatorname{Ker}(T)$, and

$$
(T \varphi, \varphi)=0=\int_{\Omega} \varepsilon\left(\left|\partial_{1} \varphi\right|^{2}+\left|\partial_{2} \varphi\right|^{2}\right)+\left(\beta^{2} \varepsilon-\omega^{2}\right)|\varphi|^{2}
$$


Since $\varepsilon \geq 1$, we have $\beta^{2} \varepsilon-\omega^{2}>0$, and thus $\varphi=\operatorname{Div}_{\beta}(\varepsilon E)=0$ in $\Omega$. Now, (5) can be written

$$
\int_{\Omega} \operatorname{Curl}_{\beta} E \cdot \overline{\operatorname{Curl}_{\beta} F}=\omega^{2} \int_{\Omega} \varepsilon E \cdot \bar{F} \quad \forall F \in V .
$$

Taking as particular test functions in (7) indefinitely differentiable fields $F$ with supports in $\Omega_{D}$ or in $\Omega_{A}$, one can check that

$$
\begin{cases}\operatorname{Curl}_{\beta} \operatorname{Curl}_{\beta} E=\omega^{2} \varepsilon E & \text { in } \Omega_{D}, \\ \operatorname{Curl}_{\beta} \operatorname{Curl}_{\beta} E=\omega^{2} \varepsilon E & \text { in } \Omega_{A} .\end{cases}
$$

The lemma will be proved if we show that $\operatorname{Curl}_{\beta} \operatorname{Curl}_{\beta} E$ belongs to $L^{2}(\Omega)^{3}$. One can easily check that this is equivalent to proving that the tangential component of $\operatorname{Curl}_{\beta} E$ is continuous through the interface $\Sigma \backslash \Gamma_{S}$ between the dielectric and the air ( $\Sigma$ denotes here the interface $\left\{x_{2}=h\right\}$ ). So let us prove that

$$
\int_{\Sigma \backslash \Gamma_{S}}\left[\left(\operatorname{Curl}_{\beta} E \times n\right) \times n\right] \overline{(F \times n)}=0 \quad \forall F \in \mathcal{C}_{0}^{\infty}(\Omega)^{3},
$$

where the integral has to be understood as a duality.

The first point to notice here is that this relation is satisfied for $F \in V$. Indeed, multiplying the two equations of (8) by $\bar{F} \in V$, one derives, thanks to Green's formula, the relation

$$
\int_{\Omega} \operatorname{Curl}_{\beta} E \cdot \overline{\operatorname{Curl}_{\beta} F}-\int_{\Sigma \backslash \Gamma_{S}}\left[\left(\operatorname{Curl}_{\beta} E \times n\right) \times n\right] \cdot \overline{(F \times n)}=\omega^{2} \int_{\Omega} \varepsilon E \cdot \bar{F} .
$$

Comparing this last equation to (7), we obtain

$$
\int_{\Sigma \backslash \Gamma_{S}}\left[\left(\operatorname{Curl}_{\beta} E \times n\right) \times n\right] \cdot \overline{(F \times n)}=0 \quad \forall F \in V .
$$

The difficulty comes now from the fact that $\mathcal{C}_{0}^{\infty}(\Omega)^{3} \not \subset V$, since $F \cdot n$ is not continuous through the interface $\Sigma \backslash \Gamma_{S}$ for $F \in V$. Nevertheless, (9) can be deduced from (10), since one can easily show that

$$
\forall F \in \mathcal{C}_{0}^{\infty}(\Omega)^{3}, \exists G=\left(G_{D}, G_{A}\right) \in V \text { such that : } G \times n=F \times n \text { on } \Sigma \backslash \Gamma_{S} .
$$

(Take, for example, $G_{A}=F$ in $\Omega_{A}$, and $G_{D}$ in $\Omega_{D}$ such that $G_{D} \times n=F \times n$ and $\varepsilon_{D} G_{D} \cdot n=\varepsilon_{A} F \cdot n$ on $\Sigma \backslash \Gamma_{S}$.)

Thus, (9) holds and, consequently,

$$
\operatorname{Curl}_{\beta} \operatorname{Curl}_{\beta} E \in L^{2}(\Omega)^{3} .
$$

Remark 2.2. (i) Notice that condition $\omega^{2}<\beta^{2}$ simply means that the mode's propagation speed is lower than the propagation speed in the vacuum. We will see later that this condition will always be satisfied if we look for eigenvalues that are not embedded in the essential spectrum.

(ii) More generally, we could have studied the regularized problem obtained by adding to the bilinear form, $\int_{\Omega} \operatorname{Curl}_{\beta} E \cdot \overline{\operatorname{Curl}_{\beta} F}$, the penalization term

$$
s \int_{\Omega} \operatorname{Div}_{\beta} E \cdot \overline{\operatorname{Div}_{\beta} F}
$$


where $s \geq 1$. The equivalence between the original problem and the regularized formulation still holds in this case, provided that the eigenvalue $\omega^{2}$ satisfies $\omega^{2}<s \beta^{2}$.

In this paper, $L^{2}(\Omega)^{3}$ is equipped with the scalar product

$$
(E, F)_{\varepsilon}=\int_{\Omega} \varepsilon E \cdot \bar{F}
$$

the associated norm being denoted

$$
\|E\|_{\varepsilon}=\left(\int_{\Omega} \varepsilon|E|^{2}\right)^{1 / 2}
$$

Let $A(\beta)$ be the unbounded operator of $L^{2}(\Omega)^{3}$ with domain $D(A(\beta))$ associated with the bilinear form $a(\beta ; \cdot, \cdot)$ defined on $V \times V$ by

$$
a(\beta, E, F)=\int_{\Omega}\left\{\operatorname{Curl}_{\beta} E \cdot \overline{\operatorname{Curl}_{\beta} F}+\operatorname{Div}_{\beta}(\varepsilon E) \overline{\operatorname{Div}_{\beta}(\varepsilon F)}\right\} .
$$

Then, problem (5) reads

For $\beta \in \mathbb{R}$, find $\omega \in \mathbb{R}$ such that there exists $E \in D(A(\beta)), E \neq 0$, satisfying

$$
A(\beta) E=\omega^{2} E .
$$

Lemma 2.1 shows that every eigenvalue $\omega^{2}$ of $A(\beta)$ such that $\omega^{2}<\beta^{2}$ corresponds to a guided mode of the microstrip line. In other words, finding the guided modes of the microstrip line amounts to the spectral analysis of the operator $A(\beta)$.

Before we achieve this spectral analysis, let us first give an explicit definition of the operator $A(\beta)$ and of its domain $D(A(\beta))$. Recall that we have the following equivalence:

$$
\left\{\begin{array} { c } 
{ E \in D ( A ( \beta ) ) } \\
{ \text { and } } \\
{ A ( \beta ) E = G }
\end{array} \Leftrightarrow \left\{\begin{array}{c}
E \in V \text { and } \exists G \in L^{2}(\Omega)^{3} \text { such that } \\
a(\beta ; E, F)=(G, F) \quad \forall F \in V .
\end{array}\right.\right.
$$

Proposition 2.3. The operator $A(\beta)$ is the operator of domain

$$
\begin{aligned}
D(A(\beta))= & \left\{E \in L^{2}(\Omega)^{3}, \operatorname{Curl}_{\beta} E \in L^{2}(\Omega)^{3}, \operatorname{Curl}_{\beta} \operatorname{Curl}_{\beta} E \in L^{2}(\Omega)^{3},\right. \\
& \left.\operatorname{Div}_{\beta}(\varepsilon E) \in H_{0}^{1}(\Omega) \text { and } E \times n_{\mid \partial \Omega}=0\right\}
\end{aligned}
$$

and such that for $E \in D(A(\beta))$

$$
A(\beta) E=\frac{1}{\varepsilon}\left\{\operatorname{Curl}_{\beta} \operatorname{Curl}_{\beta} E-\varepsilon \operatorname{Grad}_{\beta} \operatorname{Div}_{\beta}(\varepsilon E)\right\} .
$$

Proof. Set

$$
\begin{aligned}
D= & \left\{E \in L^{2}(\Omega)^{3}, \operatorname{Curl}_{\beta} E \in L^{2}(\Omega)^{3}, \operatorname{Curl}_{\beta} \operatorname{Curl}_{\beta} E \in L^{2}(\Omega)^{3},\right. \\
& \left.\operatorname{Div}_{\beta}(\varepsilon E) \in H_{0}^{1}(\Omega) \text { et } E \times n_{\mid \partial \Omega}=0\right\},
\end{aligned}
$$

and let us prove that $D=D(A(\beta))$.

(i) For $E \in D$, Green's formula shows that

$$
a(\beta ; E, F)=\int_{\Omega}\left(\operatorname{Curl}_{\beta} \operatorname{Curl}_{\beta} E-\varepsilon \operatorname{Grad}_{\beta} \operatorname{Div}_{\beta}(\varepsilon E)\right) \cdot \bar{F} d x \quad \forall F \in V .
$$


Consequently, $D \subset D(A(\beta))$ and, for $E \in D$,

$$
A(\beta) E=\frac{1}{\varepsilon}\left\{\operatorname{Curl}_{\beta} \operatorname{Curl}_{\beta} E-\varepsilon \operatorname{Grad}_{\beta} \operatorname{Div}_{\beta}(\varepsilon E)\right\} .
$$

(ii) To prove the inverse inclusion, let $E \in D(A(\beta))$ and set $G=A(\beta) E \in L^{2}(\Omega)^{3}$. Since $D(A(\beta)) \subset D(a(\beta ; \cdot, \cdot))=V$, we have

$$
\operatorname{Curl}_{\beta} E \in L^{2}(\Omega)^{3}, \operatorname{Div}_{\beta}(\varepsilon E) \in L^{2}(\Omega) \text {, and } E \times n_{\mid \partial \Omega}=0 .
$$

Moreover, thanks to Green's formula, the definition of $D(A(\beta))$ (see (12)) shows that

$$
G=A(\beta) E=\frac{1}{\varepsilon}\left\{\operatorname{Curl}_{\beta} \operatorname{Curl}_{\beta} E-\varepsilon \operatorname{Grad}_{\beta} \operatorname{Div}_{\beta}(\varepsilon E)\right\} \in L^{2}(\Omega)^{3} .
$$

Consequently, proving the inclusion $D(A(\beta)) \subset D$ amounts to showing that each of the two terms $\operatorname{Curl}_{\beta} \operatorname{Curl}_{\beta} E$ and $\operatorname{Grad}_{\beta} \operatorname{Div}_{\beta}(\varepsilon E)$ belongs to $L^{2}(\Omega)^{3}$ if $E \in D(A(\beta))$. To achieve this, the key point is to prove that the divergence $\varphi:=\operatorname{Div}_{\beta}(\varepsilon E) \in L^{2}(\Omega)$ belongs, in fact, to the space $H_{0}^{1}(\Omega)$. Notice that if we prove this result, the assertion $\operatorname{Curl}_{\beta} \operatorname{Curl}_{\beta} E \in L^{2}(\Omega)^{3}$ then follows immediately from (13).

To show that $\varphi \in H_{0}^{1}(\Omega)$, we use the same kind of arguments as those used in the proof of the equivalence between the strong formulation and the regularized variational formulation in Lemma 2.1.

Taking in (5) test functions $F$ of the form $F=\operatorname{Grad}_{\beta} \psi$, where $\psi \in H_{0}^{1}(\Omega)$ is such that $\operatorname{Div}_{\beta}\left(\varepsilon \operatorname{Grad}_{\beta} \psi\right) \in L^{2}(\Omega)^{3}$, we see that $\varphi=\operatorname{Div}_{\beta}(\varepsilon E)$ satisfies

$$
\int_{\Omega} \varphi \overline{\operatorname{Div}_{\beta}\left(\varepsilon \operatorname{Grad}_{\beta} \psi\right)}=\int_{\Omega} \varepsilon G \cdot \overline{\operatorname{Grad}_{\beta} \psi} .
$$

Now let $\tilde{\varphi} \in H_{0}^{1}(\Omega)$ be the unique solution of the coercive (for $\beta \neq 0$ ) variational problem

$$
\int_{\Omega} \varepsilon \operatorname{Grad}_{\beta} \tilde{\varphi} \cdot \overline{\operatorname{Grad}_{\beta} \psi}=-\int_{\Omega} \varepsilon G \cdot \overline{\operatorname{Grad}_{\beta} \psi} \quad \forall \psi \in H_{0}^{1}(\Omega) .
$$

Taking in (15) test functions $\psi \in H_{0}^{1}(\Omega)$ such that $\operatorname{Div}_{\beta}\left(\varepsilon \operatorname{Grad}_{\beta} \psi\right) \in L^{2}(\Omega)$, we obtain the following relation for $\tilde{\varphi}$ :

$$
\int_{\Omega} \tilde{\varphi} \overline{\operatorname{Div}_{\beta}\left(\varepsilon \operatorname{Grad}_{\beta} \psi\right)}=\int_{\Omega} \varepsilon G \cdot \overline{\operatorname{Grad}_{\beta} \psi} .
$$

Comparing this equation to (14), one can see that

$$
\int_{\Omega}(\varphi-\tilde{\varphi}) \overline{\operatorname{Div}_{\beta}\left(\varepsilon \operatorname{Grad}_{\beta} \psi\right)}=0 .
$$

The surjectivity of the operator

$$
\begin{aligned}
& T: \psi \quad \longrightarrow \quad \operatorname{Div}_{\beta}\left(\varepsilon \operatorname{Grad}_{\beta} \psi\right), \\
& H_{0}^{1}(\Omega) \quad \longrightarrow \quad L^{2}(\Omega)
\end{aligned}
$$

then implies that $\varphi=\tilde{\varphi} \in H_{0}^{1}(\Omega)$.

Proposition 2.4. The operator $A(\beta)$ is self-adjoint.

Proof. Since $A(\beta)$ is symmetric, a sufficient condition for its self-adjointness is that the operator $A(\beta)+$ Id be surjective. This follows immediately from the LaxMilgram theorem, since the bilinear form $a(\beta ; .,$.$) is clearly coercive on V \times V$.

Because the operator $A(\beta)$ is positive and self-adjoint, its spectrum is included in $\mathbb{R}^{+}$. To study its possible eigenvalues, we first determine its essential spectrum and then apply the min-max principle. 
2.4. The essential spectrum. As shown before, an important consequence of the omission of the metallic cavity is that the spectrum of $A(\beta)$ is not discrete, but involves a continuous part - the so-called essential spectrum - which corresponds to the radiating modes. The main result of this section is that this essential spectrum is identical to the essential spectrum of the unperturbed medium. Here we mean by unperturbed medium the medium obtained after removing the strip from the transmission line. Of course, this result is due to the boundedness of the strip. Nevertheless, it cannot be deduced from classical results on the spectrum's stability under compact perturbation. Indeed, the difference between the two operators involved, $A(\beta)$ for the microstrip line and $A_{\infty}(\beta)$ for the unperturbed medium, appears in their principal parts. Consequently, $A(\beta)-A_{\infty}(\beta)$ is not a compact perturbation of the operator $A_{\infty}(\beta)$.

In order to define the operator $A_{\infty}(\beta)$, let us set

$$
\varepsilon_{\infty}(x)=\varepsilon_{\infty}\left(x_{2}\right)= \begin{cases}\varepsilon_{D} & \text { if } 0<x_{2}<h \\ 1 & \text { if } x_{2}>h\end{cases}
$$

Then $A_{\infty}(\beta)$ is the operator with domain

$$
\begin{aligned}
D\left(A_{\infty}(\beta)\right)= & \left\{E \in L^{2}\left(\Omega_{\infty}\right)^{3}, \operatorname{Curl}_{\beta} E \in L^{2}\left(\Omega_{\infty}\right)^{3}, \operatorname{Curl}_{\beta} \operatorname{Curl}_{\beta} E \in L^{2}\left(\Omega_{\infty}\right)^{3},\right. \\
& \left.\operatorname{Div}_{\beta}\left(\varepsilon_{\infty} E\right) \in H_{0}^{1}\left(\Omega_{\infty}\right), \text { and } E \times n_{\mid \partial \Omega_{\infty}}=0\right\}
\end{aligned}
$$

such that

$$
A_{\infty}(\beta) E=\frac{1}{\varepsilon_{\infty}}\left\{\operatorname{Curl}_{\beta} \operatorname{Curl}_{\beta} E-\varepsilon_{\infty} \operatorname{Grad}_{\beta} \operatorname{Div}_{\beta}\left(\varepsilon_{\infty} E\right)\right\} .
$$

The operator $A_{\infty}(\beta)$ is associated with the bilinear form $a_{\infty}(\beta ; \cdot, \cdot)$, of domain

$$
\begin{aligned}
V_{\infty}= & \left\{E \in L^{2}\left(\Omega_{\infty}\right)^{3} ; \operatorname{Curl}_{\beta} E \in L^{2}\left(\Omega_{\infty}\right)^{3}, \operatorname{Div}_{\beta}\left(\varepsilon_{\infty} E\right) \in L^{2}\left(\Omega_{\infty}\right),\right. \\
& \left.E \times n_{\mid \Gamma_{G}}=0\right\}
\end{aligned}
$$

and satisfying

$$
a_{\infty}(\beta ; E, E)=\int_{\Omega_{\infty}}\left|\operatorname{Curl}_{\beta} E\right|^{2}+\left|\operatorname{Div}_{\beta}\left(\varepsilon_{\infty} E\right)\right|^{2} \quad \forall E \in V_{\infty} .
$$

We show the main result of this section in the following.

THEOREM 2.5.

$$
\sigma_{\text {ess }}(A(\beta))=\sigma_{\text {ess }}\left(A_{\infty}(\beta)\right)=\left[\gamma_{T M}(\beta),+\infty[,\right.
$$

where

$$
\gamma_{T M}(\beta)=\inf _{u \in H^{1}\left(\mathbb{R}^{+}\right), u \neq 0} \frac{\int_{0}^{+\infty} \frac{1}{\varepsilon_{\infty}\left(x_{2}\right)}\left(\left|\frac{d u}{d x_{2}}\right|^{2}+\beta^{2}|u|^{2}\right) d x_{2}}{\int_{0}^{+\infty}|u|^{2} d x_{2}} .
$$

Remark 2.6. The equation $\omega^{2}=\gamma_{T M}(\beta)$ is the dispersion relation of the fundamental transverse magnetic guided mode of the slab waveguide $(\mathrm{cf} .[7,10])$. In other 
words, $\gamma_{T M}(\beta)$ represents the first eigenvalue of the self-adjoint operator $A_{T M}(\beta)$ defined on $L^{2}\left(\mathbb{R}^{+}\right)$by

$$
\left\{\begin{array}{l}
D\left(A_{T M}(\beta)\right)=\left\{u \in H^{1}\left(\mathbb{R}^{+}\right) ; \frac{d}{d x_{2}}\left(\frac{1}{\varepsilon_{\infty}} \frac{d u}{d x_{2}}\right) \in L^{2}\left(\mathbb{R}^{+}\right), \frac{d u}{d x_{2}}(0)=0\right\}, \\
A_{T M}(\beta) u=-\frac{d}{d x_{2}}\left(\frac{1}{\varepsilon_{\infty}} \frac{d u}{d x_{2}}\right)+\frac{\beta^{2}}{\varepsilon_{\infty}} u .
\end{array}\right.
$$

One can easily check (cf. [10]) that $\gamma_{T M}(\beta)$ is the first positive solution $\gamma$ of the dispersion relation

$$
\tan (\kappa h)=\varepsilon_{D} \eta / \kappa,
$$

where we have set $\kappa=\sqrt{\gamma \varepsilon_{D}-\beta^{2}}$ and $\eta=\sqrt{\beta^{2}-\gamma}$. Furthermore, a graphical study of the dispersion equation (19) (cf. [13]) shows that $\gamma_{T M}(\beta)$ has the following properties:

$$
\left\{\begin{array}{l}
\beta \longrightarrow \gamma_{T M}(\beta) / \beta^{2} \text { is a decreasing function on } \mathbb{R}^{+}, \\
\frac{1}{\varepsilon_{D}}<\gamma_{T M}(\beta) / \beta^{2}<1 \quad \forall \beta>0, \\
\gamma_{T M}(\beta) / \beta^{2} \sim 1 \text { for } \beta \sim 0 \\
\gamma_{T M}(\beta) / \beta^{2} \sim \frac{1}{\varepsilon_{D}} \text { for } \beta \sim+\infty \\
\lim _{\beta \longrightarrow+\infty} \gamma_{T M}(\beta)-\frac{\beta^{2}}{\varepsilon_{D}}=\frac{\pi^{2}}{4 \varepsilon_{D} h^{2}} .
\end{array}\right.
$$

For the proof of Theorem 2.5, we also need to define the quantity corresponding to the fundamental transverse electric guided mode,

$$
\gamma_{T E}(\beta)=\inf _{u \in H_{0}^{1}\left(\mathbb{R}^{+}\right), u \neq 0} \frac{\int_{0}^{+\infty}\left(\left|\frac{d u}{d x_{2}}\right|^{2}+\beta^{2}|u|^{2}\right) d x_{2}}{\int_{0}^{+\infty} \varepsilon_{\infty}\left(x_{2}\right)|u|^{2} d x_{2}},
$$

which is the first positive solution $\gamma$ of the dispersion relation

$$
\tan (\kappa h)=-\kappa / \eta
$$

It can be shown that for a slab waveguide (cf. $[10,13])$, the fundamental transverse electric mode is faster than the fundamental transverse magnetic one. Indeed,

$$
0<\sqrt{\gamma_{T M}(\beta) \varepsilon_{D}-\beta^{2}}<\frac{\pi}{2 h}<\sqrt{\gamma_{T E}(\beta) \varepsilon_{D}-\beta^{2}}<\frac{\pi}{h} .
$$

Consequently,

$$
\gamma_{T M}(\beta)<\gamma_{T E}(\beta)
$$


To establish Theorem 2.5, we will demonstrate successively the following three inclusions:

$$
\begin{gathered}
\sigma_{\text {ess }}(A(\beta)) \subset \sigma_{\text {ess }}\left(A_{\infty}(\beta)\right), \quad \sigma_{\text {ess }}\left(A_{\infty}(\beta)\right) \subset\left[\gamma_{T M}(\beta),+\infty[,\right. \\
{\left[\gamma_{T M}(\beta),+\infty\left[\subset \sigma_{\text {ess }}(A(\beta)) .\right.\right.}
\end{gathered}
$$

To prove the first inclusion, we will build up a singular sequence of $A_{\infty}(\beta)$ by truncating a singular sequence of $A(\beta)$.

The main difficulty in proving the second inclusion comes from the coupling of the electric field components in $a(\beta ; E, E)$ through the curl and the divergence terms. Because the problem is invariant in the $x_{1}$-direction, we first perform a Fourier transform in this direction. Using a suitable rotation in $\left(O, x_{1}, x_{3}\right)$, we can decouple one component from the two others. The result will then follow from the properties of the transverse electric and magnetic modes of the slab waveguide.

Finally, to prove the last inclusion, we use the tools introduced to prove the second inclusion to build up for every $\lambda \geq \gamma_{T M}(\beta)$ a singular sequence of $A(\beta)$ associated with $\lambda$. Let us start by proving the first inclusion.

Proposition 2.7.

$$
\sigma_{\text {ess }}(A(\beta)) \subset \sigma_{\text {ess }}\left(A_{\infty}(\beta)\right)
$$

Proof. Let $\lambda \in \sigma_{\text {ess }}(A(\beta))$ and $E^{(p)} \in D(A(\beta))$ be a singular sequence of $A(\beta)$ associated with $\lambda$ :

$$
\left\|E^{(p)}\right\|_{\varepsilon}=1, \quad E^{(p)} \rightarrow 0 \quad \text { in } L^{2}(\Omega)^{3}, \quad\left\|A(\beta) E^{(p)}-\lambda E^{(p)}\right\|_{\varepsilon} \longrightarrow 0 .
$$

In order to build up a singular sequence of $A_{\infty}(\beta)$ associated with $\lambda$, we multiply $E^{(p)}$ by a regular function $\theta$ that vanishes in the vicinity of the strip $\Gamma_{S}$. So let us set

$$
F^{(p)}\left(x_{1}, x_{2}\right)=\theta\left(x_{1}, x_{2}\right) E^{(p)}\left(x_{1}, x_{2}\right),
$$

where $\theta \in \mathcal{C}^{\infty}\left(\Omega_{\infty}\right)$ is such that

- there exists two open sets $\mathcal{O}$ and $\mathcal{O}^{\prime}$ of $\Omega_{\infty}$, with $\Gamma_{S} \subset \mathcal{O} \subset \mathcal{O}^{\prime}$, such that

$$
\theta \equiv 0 \text { in } \mathcal{O} \text { and } \theta \equiv 1 \text { in } \Omega_{\infty} \backslash \mathcal{O}^{\prime} .
$$

- $\partial_{2} \theta \equiv 0$ in the vicinity of the interface $\Sigma=\left\{x_{2}=h\right\}$.

The sequence $F^{(p)}$ defined by (22) will be a singular sequence of $A_{\infty}(\beta)$ associated with $\lambda$ if and only if it satisfies the following properties:

(i) $\exists \delta>0,\left\|F^{(p)}\right\|_{\varepsilon_{\infty}} \geq \delta \forall p \in \mathbb{N}$.

(ii) $F^{(p)} \longrightarrow 0$ in $L^{2}\left(\Omega_{\infty}\right)^{3}$.

(iii) $F^{(p)} \in D\left(A_{\infty}(\beta)\right)$ and $\left\|A_{\infty}(\beta) F^{(p)}-\lambda F^{(p)}\right\|_{\varepsilon_{\infty}} \longrightarrow 0$.

$F^{(p)}$ clearly satisfies (i) and (ii). Indeed, we have

$$
F^{(p)}=E^{(p)}-(1-\theta) E^{(p)} \quad \text { and } \quad\left\|E^{(p)}\right\|_{\varepsilon}=1 .
$$


Since $E^{(p)}$ is bounded in $V$, the compactness of the embedding $V \subset L_{l o c}^{2}(\Omega)^{3}$ implies that

$$
(1-\theta) E^{(p)} \longrightarrow 0 \text { in } L^{2}(\Omega)^{3} .
$$

Consequently, $\left\|F^{(p)}\right\|_{L^{2}\left(\Omega_{\infty}\right)} \geq 1 / 2$ for $p$ large enough, and thus (i) is satisfied.

Property (ii) is a direct consequence of the weak convergence of $E^{(p)}$ to 0 in $L^{2}(\Omega)^{3}$.

To check property (iii), let us define for every $U=\left(U_{1}, U_{2}, U_{3}\right)^{T}$ the quantity

$$
\mathcal{A}_{\infty} U=\frac{1}{\varepsilon_{\infty}}\left\{\operatorname{Curl}_{\beta} \operatorname{Curl}_{\beta} U-\varepsilon_{\infty} \operatorname{Grad}_{\beta} \operatorname{Div}_{\beta}\left(\varepsilon_{\infty} U\right)\right\} .
$$

The important point here is that $\mathcal{A}_{\infty} F^{(p)}$ belongs to $L^{2}\left(\Omega_{\infty} \backslash \Sigma\right)$, but does not belong to $L^{2}\left(\Omega_{\infty}\right)$ (recall that $\Sigma=\left\{x_{2}=h\right\}$ ). Indeed, $F^{(p)}$ does not fulfill some of the continuity conditions on $\Sigma$, ensuring that $\mathcal{A}_{\infty} F^{(p)} \in L^{2}\left(\Omega_{\infty}\right)$. More precisely, since $\partial_{2} \theta$ vanishes in the vicinity of $\Sigma$, we have

$$
\begin{aligned}
& {\left[\operatorname{Curl}_{\beta} F^{(p)} \times n\right]_{\mid \Sigma}=-\left[E_{2}^{(p)}\right]_{\mid \Sigma}\left(\partial_{1} \theta, 0,0\right)^{T}} \\
& {\left[\operatorname{Div}_{\beta}\left(\varepsilon_{\infty} F^{(p)}\right)\right]_{\mid \Sigma}=\left[\varepsilon_{\infty} E_{1}^{(p)}\right]_{\mid \Sigma} \partial_{1} \theta}
\end{aligned}
$$

Consequently, these jumps are not (in general) equal to zero and $F^{(p)} \notin D\left(A_{\infty}(\beta)\right)$. Nevertheless, $F^{(p)}$ is a relevant choice for a singular sequence of $A_{\infty}(\beta)$ associated with $\lambda$. Indeed, a straightforward computation shows that

$$
\mathcal{A}_{\infty} F^{(p)}-\lambda F^{(p)}=\theta\left(A(\beta) E^{(p)}-\lambda E^{(p)}\right)+G^{(p)},
$$

where we have set

$$
G^{(p)}=\operatorname{grad} \theta \times \operatorname{Curl}_{\beta} E^{(p)}+\operatorname{Curl}_{\beta}\left(\operatorname{grad} \theta \times E^{(p)}\right)+\operatorname{Grad}_{\beta}\left(\operatorname{grad} \theta \cdot \varepsilon_{\infty} E^{(p)}\right) .
$$

Since $E^{(p)}$ is a singular sequence of $A(\beta)$ associated with $\lambda$, we have

$$
\theta\left(A(\beta) E^{(p)}-\lambda E^{(p)}\right) \longrightarrow 0 \text { in } L^{2}\left(\Omega_{\infty}\right)^{3} .
$$

Furthermore, classical interior regularity results for the Maxwell operator imply that $E^{(p)}$ is bounded in $H^{2}\left(\Omega_{\infty} \backslash \Sigma\right)$. Thus, since the support of $\operatorname{grad} \theta$ is included in $\mathcal{O}^{\prime} \backslash \mathcal{O}$ which is bounded, we have $G^{(p)} \longrightarrow 0$ in $L^{2}\left(\Omega_{\infty}\right)^{3}$, thanks to the compactness of the embedding of $H^{1}\left(\Omega_{\infty}\right)^{3}$ into $L_{l o c}^{2}\left(\Omega_{\infty}\right)^{3}$. Consequently, we have

$$
\mathcal{A}_{\infty} F^{(p)}-\lambda F^{(p)} \longrightarrow 0 \quad \text { in } L^{2}\left(\Omega_{\infty} \backslash \Sigma\right)^{3} .
$$

To obtain a singular sequence of $A_{\infty}(\beta)$, we need only rectify the nonzero jumps of $F^{(p)}$ across $\Sigma$. This will be achieved by adding to $F^{(p)}$ a corrective term $C^{(p)}$, defined as the solution of the boundary value problem

$$
\left\{\begin{array}{l}
\mathcal{A}_{\infty} C^{(p)}+\frac{1}{\varepsilon_{\infty}} C^{(p)}=0 \quad \text { in } \Omega_{\infty} \backslash \Sigma \\
C^{(p)} \times n_{\mid \Gamma_{P}}=0 \quad \text { and } \quad \operatorname{Div}_{\beta}\left(\varepsilon_{\infty} C^{(p)}\right)_{\mid \Gamma_{P}}=0 \\
{\left[C^{(p)} \times n\right]_{\mid \Sigma}=0 \text { and }\left[\varepsilon_{\infty} C^{(p)} \cdot n\right]_{\mid \Sigma}=0} \\
{\left[\operatorname{Curl}_{\beta} C^{(p)} \times n\right]_{\mid \Sigma}=-\left[\operatorname{Curl}_{\beta} E^{(p)} \times n\right]_{\mid \Sigma}} \\
{\left[\operatorname{Div}_{\beta}\left(\varepsilon_{\infty} C^{(p)}\right)\right]_{\mid \Sigma}=\left[\operatorname{Div}_{\beta}\left(\varepsilon_{\infty} E^{(p)}\right)\right]_{\mid \Sigma}}
\end{array}\right.
$$


The existence and uniqueness of $C^{(p)}$ follow immediately from the Lax-Milgram theorem. Furthermore, we have

$$
C^{(p)} \longrightarrow 0 \text { in } H^{1}\left(\Omega_{\infty} \backslash \Sigma\right)^{3} .
$$

Now, the sequence $F^{(p)}+C^{(p)}$ belongs to $D\left(A_{\infty}(\beta)\right)$ and still satisfies properties (i), (ii), and (iii). It is thus a singular sequence for $A_{\infty}(\beta)$ associated with $\lambda$.

Now we prove the most difficult inclusion.

PROPOSITION 2.8.

$$
\sigma_{\text {ess }}\left(A_{\infty}(\beta)\right) \subset\left[\gamma_{T M}(\beta),+\infty[.\right.
$$

Proof. Since $A_{\infty}(\beta)$ is a self-adjoint operator, the proposition will be proved if we show that

$$
\left(A_{\infty}(\beta) E, E\right)_{\varepsilon_{\infty}} \geq \gamma_{T M}(\beta)\|E\|_{\varepsilon_{\infty}}^{2} .
$$

To prove this inequality, we perform a Fourier transform in the $x_{1}$-direction and express $\left(A_{\infty}(\beta) E, E\right)_{\varepsilon_{\infty}}$ and $\|E\|_{\varepsilon_{\infty}}^{2}$ with respect to $\widehat{E}_{1}, \widehat{E}_{2}$, and $\widehat{E}_{3}$. After a rotation in the plane $\left(O, x_{1}, x_{3}\right)$, we obtain a decoupling of one component from the others. The result will then follow from the study of the monodimensional problem in $x_{2}$, obtained by "freezing" the Fourier variable. Expanding $\left(A_{\infty}(\beta) E, E\right)_{\varepsilon_{\infty}}$, we have

$$
\begin{gathered}
\left(A_{\infty}(\beta) E, E\right)_{\varepsilon_{\infty}}=\int_{\Omega_{\infty}}\left\{\left|\partial_{2} E_{3}+i \beta E_{2}\right|^{2}+\left|\partial_{1} E_{3}+i \beta E_{1}\right|^{2}+\left|\partial_{1} E_{2}-\partial_{2} E_{1}\right|^{2}\right. \\
\left.+\left|\varepsilon_{\infty} \partial_{1} E_{1}+\partial_{2}\left(\varepsilon_{\infty} E_{2}\right)-i \beta \varepsilon_{\infty} E_{3}\right|^{2}\right\} d x_{1} d x_{2} .
\end{gathered}
$$

Let us define for almost every $x_{2} \in \mathbb{R}^{+}$the Fourier transform of $E\left(\cdot, x_{2}\right) \in L^{2}(\mathbb{R})$ by

$$
\widehat{E}\left(\xi, x_{2}\right)=\frac{1}{\sqrt{2 \pi}} \int_{\mathbb{R}} E\left(x_{1}, x_{2}\right) e^{-i \xi x_{1}} d x_{1} .
$$

Then, the Plancherel theorem shows that for all $E \in D\left(A_{\infty}(\beta)\right)$

$$
\begin{aligned}
\left(A_{\infty}(\beta) E, E\right)_{\varepsilon_{\infty}}=\int_{\xi \in \mathbb{R}, x_{2} \in \mathbb{R}^{+}}\{\mid & \partial_{2} \widehat{E}_{3}+\left.i \beta \widehat{E}_{2}\right|^{2}+\left|-i \xi \widehat{E}_{3}+i \beta \widehat{E}_{1}\right|^{2} \\
& +\left|-i \xi \widehat{E}_{2}-\partial_{2} \widehat{E}_{1}\right|^{2} \\
& \left.+\left|-i \xi \varepsilon_{\infty} \widehat{E}_{1}+\partial_{2}\left(\varepsilon_{\infty} \widehat{E}_{2}\right)-i \beta \varepsilon_{\infty} \widehat{E}_{3}\right|^{2}\right\} d \xi d x_{2}
\end{aligned}
$$

and

$$
\|E\|_{\varepsilon_{\infty}}^{2}=\int_{\mathbb{R} \times \mathbb{R}^{+}} \varepsilon_{\infty}|\widehat{E}|^{2} d \xi d x_{2} .
$$

Now notice that since we have used a Fourier transform in the $x_{1}$-direction, we are looking for electromagnetic waves propagating in the direction $\mathbf{k}=(\xi / k, 0, \beta / k)$, where $k=\sqrt{\beta^{2}+\xi^{2}}$. Thus, it is natural to perform a rotation in the plane $\left(O, x_{1}, x_{3}\right)$ so that the direction defined by $\mathbf{k}$ is one of the two directions of the new system of coordinates. So, let us set

$$
\left\{\begin{array}{l}
\widehat{F}_{1}=\frac{1}{k}\left(\beta \widehat{E}_{1}-\xi \widehat{E}_{3}\right), \\
\widehat{F}_{2}=\widehat{E}_{2}, \\
\widehat{F}_{3}=\frac{1}{k}\left(\xi \widehat{E}_{1}+\beta \widehat{E}_{3}\right),
\end{array}\right.
$$


or equivalently

$$
\left\{\begin{array}{l}
\widehat{E}_{1}=\frac{1}{k}\left(\beta \widehat{F}_{1}+\xi \widehat{F}_{3}\right), \\
\widehat{E}_{2}=\widehat{F}_{2}, \\
\widehat{E}_{3}=\frac{1}{k}\left(-\xi \widehat{F}_{1}+\beta \widehat{F}_{3}\right) .
\end{array}\right.
$$

Substituting these last relations in (24), we obtain

$$
\begin{aligned}
& \left(A_{\infty}(\beta) E, E\right)_{\varepsilon_{\infty}}=\int_{\xi \in \mathbb{R}, x_{2} \in \mathbb{R}^{+}}\left\{\left|\partial_{2} \widehat{F}_{1}\right|^{2}+k^{2}\left|\widehat{F}_{1}\right|^{2}\right\} d \xi d x_{2} \\
& \quad+\int_{\xi \in \mathbb{R}, x_{2} \in \mathbb{R}^{+}}\left\{\left|\partial_{2} \widehat{F}_{3}+i k \widehat{F}_{2}\right|^{2}+\left|-i k \varepsilon_{\infty} \widehat{F}_{3}+\partial_{2}\left(\varepsilon_{\infty} \widehat{F}_{2}\right)\right|^{2}\right\} d \xi d x_{2} .
\end{aligned}
$$

Furthermore, we have $\left|\widehat{E}_{1}\right|^{2}+\left|\widehat{E}_{2}\right|^{2}+\left|\widehat{E}_{3}\right|^{2}=\left|\widehat{F}_{1}\right|^{2}+\left|\widehat{F}_{2}\right|^{2}+\left|\widehat{F}_{3}\right|^{2}$ and thus

$$
\|E\|_{\varepsilon_{\infty}}^{2}=\int_{\mathbb{R} \times \mathbb{R}^{+}} \varepsilon_{\infty}|\widehat{F}|^{2} d \xi d x_{2} .
$$

Since $\widehat{F}_{1}(\xi,.) \in H_{0}^{1}\left(\mathbb{R}^{+}\right)$for almost every $\xi \in \mathbb{R}$, the definition of $\gamma_{T E}(k)$ shows that

$$
\int_{\mathbb{R}^{+}}\left\{\left|\partial_{2} \widehat{F}_{1}\right|^{2}+k^{2}\left|\widehat{F}_{1}\right|^{2}\right\} d x_{2} \geq \gamma_{T E}(k) \int_{\mathbb{R}^{+}} \varepsilon_{\infty}\left|\widehat{F}_{1}\right|^{2} d x_{2} .
$$

Integrating this inequality with respect to $\xi$ and using the fact that $k \longrightarrow \gamma_{T E}(k)$ is a nondecreasing function, we obtain (since $k=\sqrt{\beta^{2}+\xi^{2}} \geq \beta$ )

$$
\text { (29) } \int_{\xi \in \mathbb{R}, x_{2} \in \mathbb{R}^{+}}\left\{\left|\partial_{2} \widehat{F}_{1}\right|^{2}+k^{2}\left|\widehat{F}_{1}\right|^{2}\right\} d x_{2} d \xi \geq \gamma_{T E}(\beta) \int_{\xi \in \mathbb{R}, x_{2} \in \mathbb{R}^{+}} \varepsilon_{\infty}\left|\widehat{F}_{1}\right|^{2} d x_{2} d \xi .
$$

Using the inequality (cf. Remark 2.6)

$$
\gamma_{T M}(\beta)<\gamma_{T E}(\beta)
$$

we deduce from (29) that

$$
(30) \int_{\xi \in \mathbb{R}, x_{2} \in \mathbb{R}^{+}}\left\{\left|\partial_{2} \widehat{F}_{1}\right|^{2}+k^{2}\left|\widehat{F}_{1}\right|^{2}\right\} d x_{2} d \xi \geq \gamma_{T M}(\beta) \int_{\xi \in \mathbb{R}, x_{2} \in \mathbb{R}^{+}} \varepsilon_{\infty}\left|\widehat{F}_{1}\right|^{2} d x_{2} d \xi .
$$

Integrating inequality (32) given by Lemma 2.9 below with respect to $\xi$ (notice that the fields $\widehat{F}_{2}(\xi, \cdot)$ and $\widehat{F}_{3}(\xi, \cdot)$ defined by $(25)$ belong to $\mathcal{V}$ for almost every $\left.\xi \in \mathbb{R}\right)$ and using the fact that $k \longrightarrow \gamma_{T M}(k)$ is a nondecreasing function, one obtains that

$$
\begin{aligned}
\int_{\xi \in \mathbb{R}, x_{2} \in \mathbb{R}^{+}}\left\{\left|\partial_{2} \widehat{F}_{3}+i k \widehat{F}_{2}\right|^{2}+\left|-i k \varepsilon_{\infty} \widehat{F}_{3}+\partial_{2}\left(\varepsilon_{\infty} \widehat{F}_{2}\right)\right|^{2}\right\} d \xi d x_{2} \\
\geq \gamma_{T M}(\beta) \int_{\xi \in \mathbb{R}, x_{2} \in \mathbb{R}^{+}} \varepsilon_{\infty}\left(\left|\widehat{F}_{2}\right|^{2}+\left|\widehat{F}_{3}\right|^{2}\right) d x_{2} d \xi
\end{aligned}
$$

Inequality (23), and thus Proposition 2.8, now follow immediately from the addition of inequalities (30) and (31). 
Lemma 2.9. Let $\mathcal{V}$ be the subspace of $L^{2}\left(\mathbb{R}^{+}\right) \times L^{2}\left(\mathbb{R}^{+}\right)$defined by

$$
\mathcal{V}=\left\{\widehat{F}=\left(\widehat{F}_{2}, \widehat{F}_{3}\right) \in H_{\varepsilon_{\infty}}^{1}\left(\mathbb{R}^{+}\right) \times H_{0}^{1}\left(\mathbb{R}^{+}\right)\right\},
$$

where $H_{\varepsilon_{\infty}}^{1}\left(\mathbb{R}^{+}\right)=\left\{\widehat{F}_{2} \in L^{2}\left(\mathbb{R}^{+}\right),\left(\varepsilon_{\infty} \widehat{F}_{2}\right) \in H^{1}\left(\mathbb{R}^{+}\right)\right\}$.

Then, for every $\widehat{F} \in \mathcal{V}$ and $k \geq 0$, the following inequality holds:

$$
\begin{aligned}
\int_{x_{2} \in \mathbb{R}^{+}}\left\{\left|\partial_{2} \widehat{F}_{3}+i k \widehat{F}_{2}\right|^{2}\right. & \left.+\left|-i k \varepsilon_{\infty} \widehat{F}_{3}+\partial_{2}\left(\varepsilon_{\infty} \widehat{F}_{2}\right)\right|^{2}\right\} d x_{2} \\
& \geq \gamma_{T M}(k) \int_{x_{2} \in \mathbb{R}^{+}} \varepsilon_{\infty}\left(\left|\widehat{F}_{2}\right|^{2}+\left|\widehat{F}_{3}\right|^{2}\right) d x_{2} .
\end{aligned}
$$

This lemma is proved in the appendix.

To conclude the proof of Theorem 2.5, it remains to show the following.

Proposition 2.10.

$$
\left[\gamma_{T M}(\beta),+\infty\left[\subset \sigma_{e s s}(A(\beta)) .\right.\right.
$$

Proof. First, the definition of $\gamma_{T M}(\beta)$ shows that the function $k \longrightarrow \gamma_{T M}(k)$ is bijective from $\left[\beta,+\infty\left[\right.\right.$ onto $\left[\gamma_{T M}(\beta),+\infty[\right.$. Consequently, Proposition 2.10 will be proved if we show that we can associate with every $\gamma_{T M}(k), k \geq \beta$, a singular sequence of $A(\beta)$. This singular sequence will be constructed by truncating an electric field deduced from the electric field $F$ of the slab waveguide's fundamental TM mode. One can easily check that

$$
\left\{\begin{array}{l}
F_{1}=0 \\
F_{2}=-\left(k / \omega \varepsilon_{\infty}\right) u \\
F_{3}=\left(i / \omega \varepsilon_{\infty}\right) \frac{d u}{d x_{2}}
\end{array}\right.
$$

where $u \in D\left(A_{T M}(\beta)\right)$ satisfies $A_{T M}(k) u=\gamma_{T M}(k) u$. Given a truncation function $\theta \in \mathcal{C}_{0}^{\infty}\left(\mathbb{R}^{+}\right)$, define the sequence $E^{(p)}$ by

$$
E^{(p)}\left(x_{1}, x_{2}\right)=\frac{1}{\sqrt{p}} \theta\left(x_{1} / p\right) E\left(x_{1}, x_{2}\right),
$$

where

$$
E\left(x_{1}, x_{2}\right)=\left(\begin{array}{c}
-\xi / k F_{3}\left(x_{2}\right) \\
F_{2}\left(x_{2}\right) \\
\beta / k F_{3}\left(x_{2}\right)
\end{array}\right) e^{i \xi x_{1}}
$$

Notice that, to define $E$, we have used the rotation defined by (26). Using the same arguments as those used in the proof of Proposition 2.7, we can build a singular sequence of $A(\beta)$ associated with $\lambda$ by adding to $E^{(p)}$ a corrective term that ensures the continuity conditions of the sequence across $\Sigma \backslash \Gamma_{S}$.

As already shown, we do not investigate here the question of the existence of eigenvalues $\omega^{2}$ of $A(\beta)$ embedded in the essential spectrum, i.e., such that $\omega^{2}>\gamma_{T M}(\beta)$. In the following we will find conditions for the existence of eigenvalues below the essential spectrum (these eigenvalues form the discrete spectrum). Notice furthermore that, since $\gamma_{T M}(\beta)<\beta^{2}$, every eigenvalue of $A(\beta)$ such that $\omega^{2}<\gamma_{T M}(\beta)$ corresponds to 
a guided mode. These modes propagate more slowly than the fundamental mode of the slab waveguide.

Remark 2.11. Using the same arguments as those used by Weder (cf. [17]), it could probably be shown that the possible embedded eigenvalues $\omega^{2}$ of $A(\beta)$ satisfy $\gamma_{T M}(\beta) \leq \omega^{2}<\beta^{2}$. In other words, the equivalence between the classical and the regularized formulations holds even for these eigenvalues, and they define guided modes of the microstrip line.

From now on, we denote by $\mathcal{N}(\beta)$ the number of eigenvalues $\lambda$ of $A(\beta)$ such that $\lambda<\gamma_{T M}(\beta)$ (counted with their multiplicity).

2.5. Existence of guided modes. To prove the existence of guided modes, we use the min-max principle (cf. [14]). Let $\left(\lambda_{m}(\beta)\right)_{m \geq 1}$ be the increasing sequence of positive real numbers defined as follows:

$$
\lambda_{m}(\beta)=\inf _{V_{m} \in \mathcal{V}_{m}(V)} \sup _{E \in V_{m}, E \neq 0} \frac{a(\beta ; E, E)}{\|E\|_{\varepsilon}^{2}},
$$

where $\mathcal{V}_{m}(V)$ is the set of all $m$-dimensional subspaces of $V$.

The min-max principle states that the operator $A(\beta)$ has at least $m$ eigenvalues $\lambda$ satisfying $\lambda<\gamma_{T M}(\beta)$ if and only if $\lambda_{m}(\beta)<\gamma_{T M}(\beta)$. In other words, the following assertion holds:

$$
\lambda_{m}(\beta)<\gamma_{T M}(\beta) \Longleftrightarrow \mathcal{N}(\beta) \geq m .
$$

Furthermore, if $\mathcal{N}(\beta) \geq m$, the first $m$ eigenvalues of $A(\beta)$ are exactly the numbers $\lambda_{i}(\beta), 1 \leq i \leq m$.

First, let us use this result to prove an existence result at low frequency for the fundamental mode.

TheOREM 2.12. $\mathcal{N}(\beta) \geq 1$ for $\beta$ small enough.

Proof. Inequality (34) shows that the theorem will be proved if we can establish that $\lambda_{1}(\beta)<\gamma_{T M}(\beta)$ for $\beta$ small enough, i.e., if we can find (for $\beta$ small enough) an electric field $E \in V$ such that

$$
a(\beta ; E, E)-\gamma_{T M}(\beta)\|E\|_{\varepsilon}^{2}<0 .
$$

To build such an electric field, we use the electrostatic potential, which is the solution $\varphi$ of the following boundary value problem:

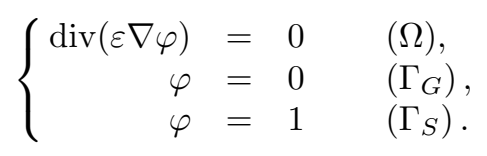

Thanks to Hardy's inequality (cf. [11]), the Lax-Milgram theorem shows that this problem has a unique solution in the weighted Sobolev space

$$
W_{0}^{1}(\Omega)=\left\{u,\left(1+|x|^{2}\right)^{-1 / 2}\left(\log \left(1+|x|^{2}\right)\right)^{-1} u(x) \in L^{2}(\Omega), \nabla u \in L^{2}(\Omega)\right\} .
$$

Then, let us set

$$
E=\left(\partial_{1} \varphi, \partial_{2} \varphi, 0\right)^{T}=(\nabla \varphi, 0) .
$$

First, we have $E \in V$. Indeed, one can easily check that

$E \in L^{2}(\Omega)^{3}, \operatorname{Div}_{\beta}(\varepsilon E)=\operatorname{div}(\varepsilon \nabla \varphi)=0$, and $\operatorname{Curl}_{\beta} E=i \beta\left(E_{2},-E_{1}, 0\right)^{T} \in L^{2}(\Omega)^{3}$. 
Furthermore, since $\varphi$ is constant on $\Gamma_{G}$ and $\Gamma_{S}$, the tangential trace of $E$ (which is nothing but the tangential derivative of $\varphi$ ) is equal to zero on $\partial \Omega=\Gamma_{G} \cup \Gamma_{S}$.

We can then compute

$$
\left\{\begin{array}{l}
a(\beta ; E, E)=\int_{\Omega}\left|\operatorname{Curl}_{\beta} E\right|^{2}=\beta^{2} \int_{\Omega}|\nabla \varphi|^{2}, \\
\|E\|_{\varepsilon}^{2}=\int_{\Omega} \varepsilon|\nabla \varphi|^{2} .
\end{array}\right.
$$

Consequently,

$$
a(\beta ; E, E)<\gamma_{T M}(\beta)\|E\|_{\varepsilon}^{2} \quad \Leftrightarrow \quad J(\varphi):=\frac{\int_{\Omega}|\nabla \varphi|^{2}}{\int_{\Omega} \varepsilon|\nabla \varphi|^{2}}<\frac{\gamma_{T M}(\beta)}{\beta^{2}} .
$$

Now notice that $\gamma_{T M}(\beta) / \beta^{2}=c_{T M}(\beta)^{2}$, where $c_{T M}(\beta)$ is the propagation speed of the fundamental TM mode of the slab waveguide. It follows from (20) that $\beta \longrightarrow c_{T M}(\beta)^{2}$ is a decreasing function on $\mathbb{R}^{+}$that satisfies

$$
\lim _{\beta \longrightarrow 0} c_{T M}(\beta)^{2}=1, \quad \quad \quad \lim _{\beta \longrightarrow+\infty} c_{T M}(\beta)^{2}=1 / \varepsilon_{D} .
$$

Since

$$
1 / \varepsilon_{D} \leq J(\varphi)=\frac{\int_{\Omega}|\nabla \varphi|^{2}}{\int_{\Omega} \varepsilon|\nabla \varphi|^{2}} \leq 1,
$$

there exists $\beta^{*}>0$ such that (36) is satisfied for $\beta<\beta^{*}$, i.e., such that the fundamental mode exists for $\beta<\beta^{*}$.

Remark 2.13. The proof of Theorem 2.12 gives an estimation of the propagation speed $c_{1}(\beta)=\left(\lambda_{1}(\beta) / \beta^{2}\right)^{1 / 2}$ of the fundamental mode. Indeed, we have

$$
c_{1}(\beta) \leq\left(\frac{a(\beta ; E, E)}{\beta^{2}\|E\|_{\varepsilon}^{2}}\right)^{1 / 2}=(J(\varphi))^{1 / 2} .
$$

Now we are going to see that, under some assumptions on the geometries of the strip and the dielectric substrate, we can establish an existence result at high frequency. Recall that $w$ and $h$ are, respectively, the width of the strip and the thickness of the dielectric substrate.

Theorem 2.14. If $\frac{w}{2 h}>m$, then $\mathcal{N}(\beta) \geq m$ for $\beta$ large enough.

Proof. Thanks to the min-max principle, this result can be obtained by proving that for $\frac{w}{2 h}>m$, the min-max $\lambda_{m}(\beta)$ (defined by (33)) satisfies $\lambda_{m}(\beta)<\gamma_{T M}(\beta)$ for $\beta$ large enough. To prove this, we first notice that for every $m$-dimensional subspace $V_{m}$ of $V$, we have from (33)

$$
\lambda_{m}(\beta) \leq \sup _{E \in V_{m}, E \neq 0} \frac{a(\beta ; E, E)}{\|E\|_{\varepsilon}^{2}} .
$$

In particular, let $V_{m}$ be the $m$-dimensional subspace of $V$ spanned by the $m$ electric fields $\left(E^{(p)}\right)_{p=1, \ldots, m}$, where

$E_{1}^{(p)}=E_{3}^{(p)}=0$ and $E_{2}^{(p)}=\left\{\begin{array}{l}\sin \left\{\frac{p \pi\left(2 x_{1}+w\right)}{2 w}\right\} \text { if } 0<x_{2}<h \text { and }\left|x_{1}\right|<\frac{w}{2}, \\ 0 \text { elsewhere. }\end{array}\right.$ 
One can easily check that $E^{(p)} \in V$ for $p=1, \ldots, m$. Furthermore, we have

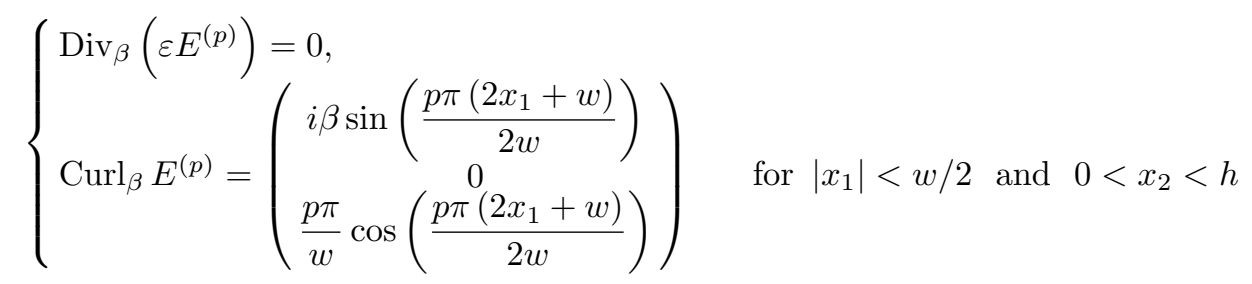

and for $1 \leq p \neq q \leq m:\left(E^{(p)}, E^{(q)}\right)=0$ and $a\left(\beta ; E^{(p)}, E^{(q)}\right)=0$. Thus

$$
\sup _{E \in V_{m}} \frac{a(\beta ; E, E)}{\|E\|_{\varepsilon}^{2}}=\frac{a\left(\beta ; E^{(m)}, E^{(m)}\right)}{\left\|E^{(m)}\right\|_{\varepsilon}^{2}} .
$$

A straightforward computation shows that

$$
\left\|E^{(m)}\right\|_{\varepsilon}^{2}=\int_{\Omega} \varepsilon\left|E^{(m)}\right|^{2}=\varepsilon_{D} \int_{-w / 2}^{w / 2} \int_{0}^{h}\left|\sin \left(\frac{m \pi\left(2 x_{1}+w\right)}{2 w}\right)\right|^{2} d x_{1} d x_{2}
$$

and

$$
a\left(\beta ; E^{(m)}, E^{(m)}\right)=\left(\beta^{2}+\frac{m^{2} \pi^{2}}{w^{2}}\right) \int_{-w / 2}^{w / 2} \int_{0}^{h}\left|\sin \left(\frac{m \pi\left(2 x_{1}+w\right)}{2 w}\right)\right|^{2} d x_{1} d x_{2} .
$$

Thus, inequality (37) reads

$$
\lambda_{m}(\beta) \leq \frac{\beta^{2}}{\varepsilon_{D}}+\frac{m^{2} \pi^{2}}{w^{2} \varepsilon_{D}} .
$$

On the other hand, we know from (20) that

$$
\lim _{\beta \longrightarrow+\infty} \gamma_{T M}(\beta)-\frac{\beta^{2}}{\varepsilon_{D}}=\frac{\pi^{2}}{4 \varepsilon_{D} h^{2}} .
$$

Consequently, if $\frac{m^{2} \pi^{2}}{w^{2} \varepsilon_{D}}<\frac{\pi^{2}}{4 \varepsilon_{D} h^{2}}$, i.e., if $\frac{w}{2 h}>m$, then for $\beta$ large enough, we have

$$
\lambda_{m}(\beta) \leq \frac{\beta^{2}}{\varepsilon_{D}}+\frac{m^{2} \pi^{2}}{w^{2} \varepsilon_{D}}<\gamma_{T M}(\beta) .
$$

Theorem 2.14 shows that at high frequency there exist at least $m$ guided modes in a perfectly conducting microstrip line if it has a sufficiently thin dielectric substrate or a sufficiently large strip.

\section{The superconducting microstrip line.}

3.1. Modelling the strip superconducting properties. The zero-thickness strip is now supposed to be made of a superconducting material. The simplest way to model the superconducting properties of a material (cf. [15]) is to use London's macroscopic model for superconductivity. It consists of adding to the classical Maxwell's equations the so-called London's equation, which relates the current $\mathbf{J}$ (in the superconductor) to the electric field $\mathbf{E}$. In the time domain, this equation reads

$$
\frac{\partial \mathbf{J}}{\partial t}=\frac{1}{\mu_{0} \lambda_{L}^{2}(T)} \mathbf{E},
$$


where $\lambda_{L}(T)$ denotes the length of London's equation, which depends only on the temperature $T$ and the nature of the superconducting material used. It represents the penetration depth of the electromagnetic field inside the superconducting material and is very small in practice. For an electromagnetic field of the form (1), London's equation becomes

$$
J=\frac{-i}{\mu_{0} \lambda_{L}^{2}(T) \omega} E
$$

which looks like Ohm's law, but with a purely imaginary conductivity, depending on the frequency $\omega$. Substituting this equation into Maxwell's equations, one can easily check that a superconducting material behaves, in London's model framework, as a material of negative dielectric permittivity $\varepsilon_{S}(\omega)$ depending on the frequency

$$
\varepsilon_{S}(\omega)=-\frac{1}{\mu_{0} \lambda_{L}^{2}(T) \omega^{2}} .
$$

Nevertheless, in the particular case of a zero-thickness strip studied here, we can use London's model to derive formally an asymptotic model (cf. [13]), in which the superconducting properties of the strip are taken into account through an impedance condition written on the strip $\Gamma_{S}$. More precisely, the asymptotic model is obtained by simultaneously letting the strip's dielectric permittivity $\varepsilon_{S}(\omega)$ tend to $-\infty$ and its thickness $d$ to zero at the same speed. In other words, we first write

$$
\varepsilon_{S}(\omega)=\varepsilon_{S}^{*}(\omega) / \delta \quad \text { and } \quad d=\delta d^{*},
$$

where $\delta$ is a small parameter and $\varepsilon_{S}^{*}(\omega)$ and $d^{*}$ are some given characteristic values. After a scaling step, we develop into a power series of delta the electromagnetic field inside the strip and look for the equations satisfied by this field as the small parameter delta tends to 0 . This leads to a model in which the tangential component of the electric field is continuous through $\Gamma_{S}$ and, moreover, proportional to the current density in the strip. In other words, the conditions satisfied by the electric field are

$$
\begin{cases}{[E \times n]=0} & \text { on }\left(\Gamma_{S}\right), \\ E \times n=\frac{1}{\Lambda}\left[\operatorname{Curl}_{\beta} E \times n\right] \times n & \text { on }\left(\Gamma_{S}\right),\end{cases}
$$

where $\Lambda$ is a positive constant.

Remark 3.1. (i) The positive constant $\Lambda$ is given by the relation

$$
\Lambda=-\mu_{0} \omega^{2} \varepsilon_{S}(\omega) d=-\mu_{0} \omega^{2} \varepsilon_{S}^{*}(\omega) d^{*} .
$$

Using (38), one should notice that (40) shows, in particular, that $\Lambda$ does not depend on the frequency, since

$$
\Lambda=d / \lambda_{L}^{2}(T)
$$

(ii) If the strip was made of a conducting material, the parameter $\Lambda$ would have been a complex number, with negative real and imaginary parts.

In the following, we will use conditions (39) to take into account the superconductivity of the strip. 
3.2. Mathematical setting of the problem. With the notation introduced in section 2.1, the equations satisfied by the electric fields corresponding to the guided modes are

$$
\begin{cases}\operatorname{Curl}_{\beta} \operatorname{Curl}_{\beta} E=\omega^{2} \varepsilon E & (\Omega) \\ \operatorname{Div}_{\beta}(\varepsilon E)=0 & (\Omega) \\ {[E \times n]=0} & \left(\Gamma_{S}\right) \\ E \times n=\frac{1}{\Lambda}\left[\operatorname{Curl}_{\beta} E \times n\right] & \left(\Gamma_{S}\right) \\ E \times n=0 & \left(\Gamma_{G}\right)\end{cases}
$$

Remark 3.2. Notice that, because of the continuity of the tangential component of $E$ across $\Gamma_{S}$, a guided mode necessarily satisfies $\operatorname{Curl}_{\beta} E \in L^{2}\left(\Omega_{\infty}\right)^{3}$.

To write a regularized variational formulation of problem (41), we proceed as in section 2.3 .

Let $W$ be the subspace of $L^{2}(\Omega)^{3}$ defined by

$$
\begin{aligned}
& W=\left\{E \in L^{2}(\Omega)^{3} ; \operatorname{Curl}_{\beta} E \in L^{2}\left(\Omega_{\infty}\right)^{3}, \operatorname{Div}_{\beta}(\varepsilon E) \in L^{2}(\Omega),\right. \\
&\left.E \times n_{\mid \Gamma_{S}} \in L^{2}\left(\Gamma_{S}\right), E \times n=0 \text { on } \Gamma_{G}\right\},
\end{aligned}
$$

and $a(\Lambda ; \beta ; \cdot, \cdot)$ the bilinear form defined on $W \times W$ by

$a(\Lambda ; \beta ; E, F)=\int_{\Omega} \operatorname{Curl}_{\beta} E \cdot \overline{\operatorname{Curl}_{\beta} F}+\int_{\Omega} \operatorname{Div}_{\beta}(\varepsilon E) \overline{\operatorname{Div}_{\beta}(\varepsilon F)}+\Lambda \int_{\Gamma_{S}}(E \times n) \cdot \overline{(F \times n)}$.

Now consider for every $\beta \in \mathbb{R}$ the following variational problem:

$$
E \in W \forall F \in W, a(\Lambda ; \beta ; E, F)=\omega^{2}(E, F)_{\varepsilon} .
$$

Using the same arguments as those used in Lemma 2.1, we obtain the following.

Lemma 3.3. Every solution of (41) satisfies (42). Conversely, if $\omega^{2}<\beta^{2}$, every solution of (42) satisfies (41).

The variational formulation (42) can be seen as a penalization of the boundary condition $E \times n=0$ on $\Gamma_{S}$, which is satisfied in the case of the perfectly conducting strip studied in section 2. One can also formally notice from (39) that this case corresponds to the limit problem obtained for $\Lambda$ tending to infinity. This result will be rigorously proved in section 3.4.

Before studying the spectral properties of the operator associated with the bilinear form $a(\Lambda ; \beta ; \cdot, \cdot)$, let us point out some useful properties of its domain $W$. First, as can easily be checked from its definition, $W$ does not depend on $\beta$. More precisely, we have

$$
\begin{aligned}
W= & \left\{E \in L^{2}(\Omega)^{3} ; E_{3} \in H^{1}(\Omega), E_{2,1}-E_{1,2} \in L^{2}(\Omega), \varepsilon E_{1,1}+\left(\varepsilon E_{2}\right)_{, 2} \in L^{2}(\Omega),\right. \\
& {\left.[E \times n]=0 \text { on } \Gamma_{S}, E \times n_{\mid \Gamma_{S}} \in L^{2}\left(\Gamma_{S}\right), E \times n=0 \text { on } \Gamma_{G}\right\} }
\end{aligned}
$$

Furthermore, $W$ is a Hilbert space for the norm

$$
\|E\|_{W}=\sqrt{\|E\|_{\varepsilon}^{2}+\left\|\operatorname{Curl}_{\beta} E\right\|_{\varepsilon}^{2}+\left\|\operatorname{Div}_{\beta} E\right\|_{\varepsilon}^{2}+\|E \times n\|_{0, \Gamma_{S}}^{2}},
$$

and the embedding of $W$ in $L_{l o c}^{2}(\Omega)^{3}$ is compact (cf. [8]). 
Finally, one should notice that $V \subset W$ (where $V$ is defined by (4)), since the conditions $[E \times n]_{\mid \Gamma_{S}}=0$ and $E \times n \in L^{2}\left(\Gamma_{S}\right)$ are clearly fulfilled by the electric fields $E$ that belong to $V$. As we will see later, this property will be very useful to establish existence results for the guided modes.

Because the space $L^{2}(\Omega)^{3}$ is equipped with the scalar product $(\cdot, \cdot)_{\varepsilon}$ defined by (11), let $A(\Lambda ; \beta)$ be the unbounded operator of $L^{2}(\Omega)^{3}$ associated with the bilinear form $a(\Lambda ; \beta, \cdot, \cdot)$.

Finding guided modes of the microstrip line amounts to finding the positive eigenvalues of $A(\Lambda ; \beta)$ such that $\omega^{2}<\beta^{2}$. Since $A(\Lambda ; \beta)$ is a positive self-adjoint operator, its spectrum is included in $\mathbb{R}^{+}$and is composed of a (possibly empty) discrete part and a continuous part. Before using the min-max principle to show existence results of guided modes, we have to determine the essential spectrum $\sigma_{\text {ess }}(A(\Lambda ; \beta))$ of $A(\Lambda ; \beta)$. Using the same arguments as those used to prove Theorem 2.5 , we can establish that this spectrum is still the same as that of the unperturbed medium.

THEOREM 3.4.

$$
\sigma_{e s s}(A(\Lambda ; \beta))=\left[\gamma_{T M}(\beta),+\infty[.\right.
$$

Remark 3.5. (i) If we had used a conducting strip instead of a superconducting one (see Remark 3.1), the bilinear form $a(\Lambda ; \beta ; \cdot, \cdot)$ obtained would still have been coercive, although $\operatorname{Re}(\Lambda)<0$. This result is a direct consequence of the fact that $\Lambda$ has a nonzero imaginary part. Nevertheless, this last property is also responsible for the loss of symmetry of $a(\Lambda ; \beta ; \cdot, \cdot)$. Consequently, the operator $A(\Lambda ; \beta)$ is not self-adjoint in this case, and its spectral analysis is much more complicated.

(ii) As already shown, a superconducting and nonzero-thickness strip behaves as a material of negative dielectric permittivity, depending on the frequency. The change of sign of the permittivity (positive outside the strip and negative inside) makes the operator involved no longer elliptic and its self-adjointness study quite difficult.

3.3. Existence of guided modes. The results presented here follow from those obtained in section 2.5 in the case of the perfectly conducting microstrip line. Let $\mathcal{N}(\beta)$ (respectively, $\mathcal{N}(\Lambda ; \beta)$ ) be the number of guided modes of this line (respectively, of the superconducting microstrip line studied in this section). Recall that $\mathcal{N}(\beta)$ and $\mathcal{N}(\Lambda ; \beta)$ are, respectively, the numbers of eigenvalues $\lambda$ of $A(\beta)$ and $A(\Lambda ; \beta)$ such that $\lambda<\gamma_{T M}(\beta)$, counted with their order of multiplicity. Then we have the following.

THEOREM 3.6. For fixed $\beta, \Lambda \longrightarrow \mathcal{N}(\Lambda ; \beta)$ is a decreasing function which is bounded from below by $\mathcal{N}(\beta)$ :

$$
\mathcal{N}(\Lambda ; \beta) \geq \mathcal{N}(\beta) .
$$

Proof. The key point of the proof is the fact that $V \subset W$ and that for every $E \in V$, we have

$$
a(\Lambda ; \beta ; E, E)=a(\beta ; E, E) .
$$

Consequently,

$$
\inf _{V_{m} \in \mathcal{V}_{m}(V)} \sup _{E \in V_{m}, E \neq 0} \frac{a(\beta ; E, E)}{\|E\|_{\varepsilon}^{2}}=\inf _{V_{m} \in \mathcal{V}_{m}(V)} \sup _{E \in V_{m}, E \neq 0} \frac{a(\Lambda ; \beta ; E, E)}{\|E\|_{\varepsilon}^{2}}
$$

and

$$
\inf _{V_{m} \in \mathcal{V}_{m}(V)} \sup _{E \in V_{m}, E \neq 0} \frac{a(\Lambda ; \beta ; E, E)}{\|E\|_{\varepsilon}^{2}} \geq \inf _{W_{m} \in \mathcal{V}_{m}(W)} \sup _{E \in W_{m}, E \neq 0} \frac{a(\Lambda ; \beta ; E, E)}{\|E\|_{\varepsilon}^{2}} .
$$


In other words, we have shown that

$$
\lambda_{m}(\beta) \geq \lambda_{m}(\Lambda ; \beta) .
$$

The min-max principle then shows that

$$
\mathcal{N}(\Lambda ; \beta) \geq \mathcal{N}(\beta)
$$

The fact that the function $\Lambda \longrightarrow \mathcal{N}(\Lambda ; \beta)$ is decreasing also follows from the min-max principle, since $\Lambda \longrightarrow \lambda_{m}(\Lambda ; \beta)$ is clearly a nondecreasing function for fixed $\beta$.

Thanks to result (i) of Theorem 3.6, it immediately follows from Theorems 2.12 and 2.14 that the same existence results as those obtained for a perfectly conducting strip hold.

THEOREM 3.7.

(i) $\mathcal{N}(\Lambda ; \beta) \geq 1$ for $\beta$ small enough.

(ii) If $\frac{w}{2 h}>m$, then $\mathcal{N}(\Lambda ; \beta) \geq m$ for $\beta$ large enough.

3.4. Asymptotic behavior of the guided modes for $\Lambda \longrightarrow+\infty$. Problem (41) can be seen as a penalization of the problem corresponding to a perfectly conducting strip of zero thickness, the penalization parameter being $\Lambda$. In this section, we make this statement precise by proving that the fundamental guided mode and the corresponding eigenvalue of $A(\Lambda ; \beta)$ converge, as $\Lambda \longrightarrow+\infty$, to the corresponding eigenelements of $A(\beta)$. This result is given by the following.

Theorem 3.8. Suppose that $\mathcal{N}(\beta) \geq 1$ and let $\lambda_{1}(\Lambda ; \beta)$ (respectively, $\lambda_{1}(\beta)$ ) be the first eigenvalue of the operator $A(\Lambda ; \beta)$ (respectively, $A(\beta)$ ). Then

$$
\lim _{\Lambda \longrightarrow+\infty} \lambda_{1}(\Lambda ; \beta)=\lambda_{1}(\beta) .
$$

Furthermore, if $E(\Lambda ; \beta) \in W$ denotes an eigenvector associated with $\lambda_{1}(\Lambda ; \beta)$, then there exist a subsequence of $E(\Lambda ; \beta)$, still denoted $E(\Lambda ; \beta)$, and an eigenvector $E(\beta) \in$ $V$ of $A(\beta)$ associated with $\lambda_{1}(\beta)$ such that

$$
E(\Lambda ; \beta) \longrightarrow E(\beta) \quad \text { in } W .
$$

Proof. To make the proof easier to read, we will omit in the notation the dependence with respect to $\beta$. For instance, the operator $A(\Lambda ; \beta)$ (respectively, $A(\beta)$ ) is denoted $A(\Lambda)$ (respectively, $A$ ).

First, let us notice that since $\mathcal{N}(\Lambda) \geq \mathcal{N} \geq 1$ (see Theorem 3.6), the first eigenvalue $\lambda_{1}(\Lambda)$ of $A(\Lambda)$ exists.

In order to establish Theorem 3.8, we are going to prove successively the following assertions:

(i) There exist a real number $\lambda_{1}^{*}$ and an electric field $E \in V$ such that

$$
\lim _{\Lambda \longrightarrow+\infty} \lambda_{1}(\Lambda)=\lambda_{1}^{*} \quad \text { and } \quad E(\Lambda) \rightarrow E \text { weakly in } W .
$$

(ii) $E(\Lambda) \longrightarrow E$ strongly in $W$.

(iii) $\lambda_{1}^{*}=\lambda_{1}$ and $E$ is an eigenvector of $A$ associated with $\lambda_{1}$.

Let us start by proving property (i).

(i) The function $\Lambda \longrightarrow \lambda_{1}(\Lambda)$ is nondecreasing and satisfies $\lambda_{1}(\Lambda) \leq \lambda_{1}$ (see (43)). Thus, there exist $\lambda_{1}^{*}=\lim _{\Lambda \longrightarrow+\infty} \lambda_{1}(\Lambda)$, and, furthermore, we have

$$
\lambda_{1}^{*} \leq \lambda_{1} .
$$


Let $E(\Lambda)$ be an eigenvector associated with $\lambda_{1}(\Lambda)$ satisfying (without loss of generality) the condition $\|E(\Lambda)\|_{\varepsilon}=1$. Then

$$
a(\Lambda ; E(\Lambda), E(\Lambda))=\lambda_{1}(\Lambda) .
$$

This equation can be written

$$
\int_{\Omega}\left|\operatorname{Curl}_{\beta} E(\Lambda)\right|^{2}+\left|\operatorname{Div}_{\beta}(\varepsilon E(\Lambda))\right|^{2}+\Lambda \int_{\Gamma_{S}}|E(\Lambda) \times n|^{2}=\lambda_{1}(\Lambda) .
$$

Consequently, $E(\Lambda)$ is bounded in $W$ (with respect to $\Lambda$ ), and there exist $E$ in $W$ and a subsequence of $E(\Lambda)$, still denoted $E(\Lambda)$, such that $E(\Lambda) \rightarrow E$ in $W$. Furthermore, since (45) implies that

$$
\lim _{\Lambda \longrightarrow+\infty} \int_{\Gamma_{S}}|E(\Lambda) \times n|^{2}=0,
$$

we have $E \times n_{\mid \Gamma_{S}}=0$ and $E$ belongs to $V$.

(ii) To prove that $e(\Lambda)=E(\Lambda)-E$ converges to 0 in $W$, we shall first prove that it converges to 0 in $L^{2}(\Omega)^{3}$.

Taking the limit as $\Lambda \longrightarrow+\infty$ in the variational formulation (42) satisfied for every $F \in V \subset W$, one obtains that

$$
a(\Lambda ; E, F)=a(E, F)=\lambda_{1}^{*}(E, F)_{\varepsilon} \quad \forall F \in V .
$$

It then follows from (42) and (47) that

$$
a(\Lambda ; e(\Lambda), e(\Lambda))=\lambda_{1}(\Lambda)+\lambda_{1}^{*}\|E\|_{\varepsilon}^{2}-2 \lambda_{1}(\Lambda)(E(\Lambda), E)_{\varepsilon} .
$$

Since $\|e(\Lambda)\|_{\varepsilon}^{2}=1+\|E\|_{\varepsilon}^{2}-2(E(\Lambda), E)$, (48) shows that

$$
a(\Lambda ; e(\Lambda), e(\Lambda))=\lambda_{1}(\Lambda)\|e(\Lambda)\|_{\varepsilon}^{2}+\left(\lambda_{1}^{*}-\lambda_{1}(\Lambda)\right)\|E\|_{\varepsilon}^{2} .
$$

Now set

$$
e_{\infty}(\Lambda)=\theta e(\Lambda)
$$

where $\theta \in \mathcal{C}^{\infty}\left(\Omega_{\infty}\right)$ satisfies

- $\forall x \in \Omega_{\infty}: 0 \leq \theta(x) \leq 1$.

- There exist two open sets $\mathcal{O}$ and $\mathcal{O}^{\prime}$ of $\Omega$ such that

$$
\left\{\begin{array}{l}
\Gamma_{S} \subset \mathcal{O} \subset \mathcal{O}^{\prime}, \\
\theta=0 \quad \text { in } \mathcal{O}, \\
\theta=1 \quad \text { in } \Omega_{\infty} \backslash \mathcal{O}^{\prime} .
\end{array}\right.
$$

- $\theta\left(x_{1}, x_{2}\right)=\theta\left(x_{1}\right)$ in the vicinity of the interface $\Sigma=\left\{x_{2}=h\right\}$ between the dielectric substrate and the air.

It is clear that $e_{\infty}(\Lambda) \in V_{\infty}$, where $V_{\infty}$ (defined by (16)) is the domain of the bilinear form $a_{\infty}(\cdot, \cdot)$ (defined by $\left.(17)\right)$ associated with $A_{\infty}$. Since

$$
\gamma_{T M}=\inf \left(\sigma_{e s s}\left(A_{\infty}\right)\right)=\inf _{F \in V_{\infty}, F \neq 0} \frac{a_{\infty}(F, F)}{\|F\|_{\varepsilon_{\infty}}^{2}},
$$


we have

$$
a_{\infty}\left(e_{\infty}(\Lambda), e_{\infty}(\Lambda)\right) \geq \gamma_{T M}\left\|e_{\infty}(\Lambda)\right\|_{\varepsilon_{\infty}}^{2} .
$$

A straightforward computation shows that

$$
\left\{\begin{array}{l}
a_{\infty}\left(e_{\infty}(\Lambda), e_{\infty}(\Lambda)\right)=\int_{\Omega}|\theta|^{2}\left\{\left|\operatorname{Curl}_{\beta} e(\Lambda)\right|^{2}+\left|\operatorname{Div}_{\beta}(\varepsilon e(\Lambda))\right|^{2}\right\}+G(\Lambda) \\
\left\|e_{\infty}(\Lambda)\right\|_{\varepsilon_{\infty}}^{2}=\|e(\Lambda)\|_{\varepsilon}^{2}+H(\Lambda),
\end{array}\right.
$$

where

$$
\left\{\begin{aligned}
G(\Lambda)= & \int_{\Omega}|\nabla \theta \times e(\Lambda)|^{2}+2 \operatorname{Re}\left(\int_{\Omega}\left(\theta \operatorname{Curl}_{\beta} e(\Lambda)\right) \cdot \overline{(\nabla \theta \times e(\Lambda))}\right) \\
& +\int_{\Omega}|\nabla \theta \cdot \varepsilon e(\Lambda)|^{2}+2 \operatorname{Re}\left(\int_{\Omega}\left(\theta \operatorname{Div}_{\beta}(\varepsilon e(\Lambda))\right) \cdot \overline{(\nabla \theta \cdot e(\Lambda))}\right), \\
H(\Lambda)= & -\int_{\Omega} \varepsilon\left(1-\theta^{2}\right)|e(\Lambda)|^{2} .
\end{aligned}\right.
$$

Inequality (50) then reads

$$
\int_{\Omega}|\theta|^{2}\left(\left|\operatorname{Curl}_{\beta} e(\Lambda)\right|^{2}+\left|\operatorname{Div}_{\beta}(\varepsilon e(\Lambda))\right|^{2}\right) \geq \gamma_{T M}\|e(\Lambda)\|_{\varepsilon}^{2}+\left(\gamma_{T M} H(\Lambda)-G(\Lambda)\right) .
$$

Since $0 \leq \theta \leq 1$, this inequality implies that

$$
a(\Lambda ; e(\Lambda), e(\Lambda)) \geq \gamma_{T M}\|e(\Lambda)\|_{\varepsilon}^{2}+\left(\gamma_{T M} H(\Lambda)-G(\Lambda)\right) .
$$

We now deduce from (49) and (51) that

$$
\left(\gamma_{T M}-\lambda_{1}(\Lambda)\right)\|e(\Lambda)\|_{\varepsilon}^{2} \leq\left(\lambda_{1}^{*}-\lambda_{1}(\Lambda)\right)\|E\|_{\varepsilon}^{2}-\left(\gamma_{T M} H(\Lambda)-G(\Lambda)\right) .
$$

To conclude the proof of (ii), we notice that (thanks to the compactness of the embedding of $W$ in $\left.L_{l o c}^{2}(\Omega)^{3}\right)$

$$
\left\{\begin{array}{l}
e(\Lambda) \longrightarrow 0 \quad \text { in } L_{l o c}^{2}(\Omega)^{3}, \\
\left\|\operatorname{Curl}_{\beta} e(\Lambda)\right\|_{\varepsilon} \quad \text { and } \quad\left\|\operatorname{Div}_{\beta}(\varepsilon e(\Lambda))\right\|_{\varepsilon} \quad \text { are bounded, } \\
(1-\theta) \text { and } \nabla \theta \text { have compact supports. }
\end{array}\right.
$$

Thus

$$
\lim _{\Lambda \longrightarrow+\infty} G(\Lambda)=\lim _{\Lambda \longrightarrow+\infty} H(\Lambda)=0 .
$$

On the other hand, we have

$$
\gamma_{T M}-\lambda_{1}(\Lambda) \geq \gamma_{T M}-\lambda_{1}>0 \quad \text { and } \quad \quad \Lambda \stackrel{\lim _{\longrightarrow}}{\longrightarrow} \lambda_{1}(\Lambda)=\lambda_{1}^{*} .
$$

Consequently, (52) implies that

$$
\lim _{\Lambda \longrightarrow+\infty}\|e(\Lambda)\|_{\varepsilon}^{2}=\lim _{\Lambda \longrightarrow+\infty}\|E(\Lambda)-E\|_{\varepsilon}^{2}=0 .
$$


The convergence in $W$ now follows from the fact that (for $\Lambda \geq 1$ )

$$
\|E(\Lambda)-E\|_{W}=\|e(\Lambda)\|_{W} \leq a(\Lambda ; e(\Lambda), e(\Lambda))=\lambda_{1}(\Lambda)\|e(\Lambda)\|_{\varepsilon}^{2}+\left(\lambda_{1}^{*}-\lambda_{1}(\Lambda)\right)\|E\|_{\varepsilon}^{2} .
$$

(iii) Since $E(\Lambda)-E$ converges to 0 in $L^{2}(\Omega)^{3}$ and $\|E(\lambda)\|_{\varepsilon}=1$, we have $E \neq 0$. Furthermore, (47) shows that

$$
\lambda_{1}^{*}=\frac{a(E, E)}{\|E\|_{\varepsilon}^{2}} .
$$

On the other hand, the min-max principle shows that

$$
\lambda_{1}=\inf _{F \in V, F \neq 0} \frac{a(F, F)}{\|F\|_{\varepsilon}^{2}} .
$$

Consequently,

$$
\lambda_{1} \leq \lambda_{1}^{*}
$$

Joined to (44), this inequality implies that

$$
\lambda_{1}=\lambda_{1}^{*},
$$

and thus $E$ is an eigenvector associated with $\lambda_{1}$.

Remark 3.9. Using the same arguments, it can be proved that the results of Theorem 3.8 may be extended to the guided modes of higher order. In other words, if $\mathcal{N}(\beta) \geq m$, we have (with obvious notation)

$$
\lim _{\Lambda \longrightarrow+\infty} \lambda_{m}(\Lambda ; \beta)=\lambda_{m}(\beta) \quad \text { and } \quad E_{m}(\Lambda ; \beta) \longrightarrow E_{m}(\beta) \text { in } W .
$$

Appendix. This appendix is devoted to the proof of Lemma 2.9. We have to show that the following inequality holds for every $\widehat{F}=\left(\widehat{F}_{2}, \widehat{F}_{3}\right) \in \mathcal{V}$ :

$$
\begin{aligned}
\int_{x_{2} \in \mathbb{R}^{+}}\left\{\left|\partial_{2} \widehat{F}_{3}+i k \widehat{F}_{2}\right|^{2}\right. & \left.+\left|-i k \varepsilon_{\infty} \widehat{F}_{3}+\partial_{2}\left(\varepsilon_{\infty} \widehat{F}_{2}\right)\right|^{2}\right\} d x_{2} \\
& \geq \gamma_{T M}(k) \int_{x_{2} \in \mathbb{R}^{+}} \varepsilon_{\infty}\left(\left|\widehat{F}_{2}\right|^{2}+\left|\widehat{F}_{3}\right|^{2}\right) d x_{2},
\end{aligned}
$$

where

$$
\mathcal{V}=\left\{\widehat{F}=\left(\widehat{F}_{2}, \widehat{F}_{3}\right) \in H_{\varepsilon_{\infty}}^{1}\left(\mathbb{R}^{+}\right) \times H_{0}^{1}\left(\mathbb{R}^{+}\right)\right\}
$$

and

$$
H_{\varepsilon_{\infty}}^{1}\left(\mathbb{R}^{+}\right)=\left\{\widehat{F}_{2} \in L^{2}\left(\mathbb{R}^{+}\right),\left(\varepsilon_{\infty} \widehat{F}_{2}\right) \in H^{1}\left(\mathbb{R}^{+}\right)\right\}
$$

Proof. The main difficulty comes from the coupling of the two components $\widehat{F}_{2}$ and $\widehat{F}_{3}$, which is induced by the regularizing term. 
Consider the bilinear form $b_{\infty}(\beta ; .,$.$) defined for (\widehat{F}, \widehat{G}) \in \mathcal{V} \times \mathcal{V}$ by

$$
\begin{aligned}
b_{\infty}(k ; \widehat{F}, \widehat{G})= & \int_{\mathbb{R}^{+}}\left(\frac{d \widehat{F}_{3}}{d x_{2}}+i k \widehat{F}_{2}\right) \overline{\left(\frac{d \widehat{G}_{3}}{d x_{2}}+i k \widehat{G}_{2}\right)} \\
& +\left(-i k \varepsilon_{\infty} \widehat{F}_{3}+\frac{d\left(\varepsilon_{\infty} \widehat{F}_{2}\right)}{d x_{2}}\right) \overline{\left(-i k \varepsilon_{\infty} \widehat{G}_{3}+\frac{d\left(\varepsilon_{\infty} \widehat{G}_{2}\right)}{d x_{2}}\right)} d x_{2} .
\end{aligned}
$$

The lemma will be proved if the following inequality holds:

$$
b_{\infty}(k ; \widehat{F}, \widehat{F}) \geq \gamma_{T M}(k)\|\widehat{F}\|_{\varepsilon_{\infty}}^{2} \quad \forall \widehat{F} \in \mathcal{V},
$$

where

$$
\|\widehat{F}\|_{\varepsilon_{\infty}}^{2}=\int_{\mathbb{R}^{+}} \varepsilon_{\infty}|\widehat{F}|^{2} d x_{2}=\int_{\mathbb{R}^{+}} \varepsilon_{\infty}\left(\left|\widehat{F}_{2}\right|^{2}+\left|\widehat{F}_{3}\right|^{2}\right) d x_{2}
$$

Indeed, inequality (53) can immediately be obtained by integrating inequality (54), with respect to $\xi$, and using the fact that $t \longrightarrow \gamma_{T M}(t)$ is a nondecreasing function.

So let us prove $(54)$.

Let $\widehat{F} \in \mathcal{V}$, and define $\widehat{\varphi}$ as the unique function of $H_{0}^{1}\left(\mathbb{R}^{+}\right)$such that

$$
-\frac{d}{d x_{2}}\left(\varepsilon_{\infty} \frac{d \widehat{\varphi}}{d x_{2}}\right)+k^{2} \varepsilon_{\infty} \widehat{\varphi}=\frac{d}{d x_{2}}\left(\varepsilon_{\infty} \widehat{F}_{2}\right)-i k \varepsilon_{\infty} \widehat{F}_{3}
$$

Then, if we set

$$
\widehat{G}=\widehat{F}+\left(\frac{d \widehat{\varphi}}{d x_{2}},-i k \widehat{\varphi}\right)
$$

we have $\widehat{G} \in \mathcal{V}$, and thanks to (55),

$$
\frac{d}{d x_{2}}\left(\varepsilon_{\infty} \widehat{G}_{2}\right)-i k \varepsilon_{\infty} \widehat{G}_{3}=0 .
$$

A straightforward computation then shows that

$$
b_{\infty}(k ; \widehat{F}, \widehat{F})=\int_{\mathbb{R}^{+}}\left|\frac{d \widehat{G}_{3}}{d x_{2}}+i k \widehat{G}_{2}\right|^{2}+\int_{\mathbb{R}^{+}}\left|-\frac{d}{d x_{2}}\left(\varepsilon_{\infty} \frac{d \widehat{\varphi}}{d x_{2}}\right)+k^{2} \varepsilon_{\infty} \widehat{\varphi}\right|^{2} d x_{2}
$$

and

$$
\|\widehat{F}\|_{\varepsilon_{\infty}}^{2}=\int_{\mathbb{R}^{+}} \varepsilon_{\infty}|\widehat{G}|^{2}+\int_{\mathbb{R}^{+}} \varepsilon_{\infty}\left(\left|\frac{d \widehat{\varphi}}{d x_{2}}\right|^{2}+k^{2}|\widehat{\varphi}|^{2}\right) .
$$

Consequently, to prove (54) it is sufficient to prove the following two inequalities:

$$
\int_{\mathbb{R}^{+}}\left|\frac{d \widehat{G}_{3}}{d x_{2}}+i k \widehat{G}_{2}\right|^{2} \geq \gamma_{T M}(k) \int_{\mathbb{R}^{+}} \varepsilon_{\infty}|\widehat{G}|^{2}
$$


and

(58) $\int_{\mathbb{R}^{+}}\left|-\frac{d}{d x_{2}}\left(\varepsilon_{\infty} \frac{d \widehat{\varphi}}{d x_{2}}\right)+k^{2} \varepsilon_{\infty} \widehat{\varphi}\right|^{2} d x_{2} \geq \gamma_{T M}(k) \int_{\mathbb{R}^{+}} \varepsilon_{\infty}\left(\left|\frac{d \widehat{\varphi}}{d x_{2}}\right|^{2}+k^{2}|\widehat{\varphi}|^{2}\right)$.

Let us start by proving (57). First, notice that (56) shows the existence of $\widehat{\psi} \in$ $D\left(A_{T M}(k)\right)$ such that

$$
\left\{\begin{array}{l}
\widehat{G}_{2}=\frac{i k}{\varepsilon_{\infty}} \widehat{\psi} \\
\widehat{G}_{3}=\frac{1}{\varepsilon_{\infty}} \frac{d \widehat{\psi}}{d x_{2}}
\end{array}\right.
$$

Inequality (57) then reads, in terms of $\widehat{\psi}$,

$(59) \int_{\mathbb{R}^{+}} \mid-\frac{d}{d x_{2}}\left(\frac{1}{\varepsilon_{\infty}} \frac{d \widehat{\psi}}{d x_{2}}\right)+\frac{k^{2}}{\varepsilon_{\infty}} \widehat{\psi}^{2} d x_{2} \geq \gamma_{T M}(k) \int_{\mathbb{R}^{+}} \frac{1}{\varepsilon_{\infty}}\left(\left|\frac{d \widehat{\psi}}{d x_{2}}\right|^{2}+k^{2}|\widehat{\psi}|^{2}\right)$,

or equivalently,

$$
\left\|A_{T M}(k) \widehat{\psi}\right\|_{L^{2}}^{2} \geq \gamma_{T M}(k)\left(A_{T M}(k) \widehat{\psi}, \widehat{\psi}\right)_{L^{2}},
$$

where we have set $(u, v)_{L^{2}}=\int_{\mathbb{R}^{+}} u \bar{v}$ and $\|u\|_{L^{2}}^{2}=\int_{\mathbb{R}^{+}}|u|^{2}$.

Setting $\widehat{f}=A_{T M}(k) \widehat{\psi}$, inequality (60) can be written

$$
\frac{\left(A_{T M}(k)^{-1} \widehat{f}, \widehat{f}\right)_{L^{2}}}{\|\hat{f}\|_{L^{2}}^{2}} \leq \frac{1}{\gamma_{T M}(k)} .
$$

Since the essential spectrum of the self-adjoint operator $A_{T M}(k)^{-1}$ is the interval $\left[0,1 / k^{2}\right]$, and $1 / \gamma_{T M}(k)$ is nothing but the largest eigenvalue of $A_{T M}(k)^{-1}$, we have

$$
\frac{\left(A_{T M}(k)^{-1} \widehat{f}, \widehat{f}\right)_{L^{2}}}{\|\widehat{f}\|_{L^{2}}^{2}} \leq \sup \sigma\left(A_{T M}(k)^{-1}\right)=\frac{1}{\gamma_{T M}(k)}
$$

and (61) - and thus (57) - is satisfied.

Comparing (58) and (59), we see that (58) can be proved simply by replacing $\varepsilon_{\infty}$ with $1 / \varepsilon_{\infty}$ in the previous arguments. In this case, the spectrum of the operator $A_{T M}(k)^{-1}$ is purely continuous and is still equal to $\left[0,1 / k^{2}\right]$. Thus

$$
\sup \sigma\left(A_{T M}(k)^{-1}\right)=1 / k^{2} \leq 1 / \gamma_{T M}(k),
$$

and consequently,

$$
\left\|A_{T M}(k) \widehat{\varphi}\right\|_{L^{2}}^{2} \geq \gamma_{T M}(k)\left(A_{T M}(k) \widehat{\varphi}, \widehat{\varphi}\right)_{L^{2}} .
$$




\section{REFERENCES}

[1] A. Bamberger And A.-S. Bonnet, Mathematical analysis of the guided modes of an optical fiber, SIAM J. Math. Anal., 21 (1990), pp. 1487-1510.

[2] A.-S. Bonnet-Ben Dhia, G. Caloz, And F. Mahé, Guided modes of integrated optical guides. A mathematical study, IMA J. Appl. Math., 60 (1998), pp. 225-261.

[3] A.-S. Bonnet-Ben Dhia, J. Duterte, And P. Joly, Mathematical analysis of elastic surface waves in topographic waveguides, Math. Models Methods Appl. Sci., 9 (1999), pp. 755-798.

[4] A.-S. Bonnet-Ben Dhia, C. Hazard, and S. Lohrengel, A singular field method for the solution of Maxwell's equations in polyhedral domains, SIAM J. Appl. Math., 59 (1999), pp. 2028-2044.

[5] A.-S. Bonnet-Ben Dhia And P. Joly, Mathematical analysis of guided water waves, SIAM J. Appl. Math., 53 (1993), pp. 1507-1550.

[6] R. E. Collins, Foundations for Microwave Engineering, McGraw-Hill Series in Electrical Engineering. Radar and Antennas, McGraw-Hill, New York, 1992.

[7] J. C. Guillot, Complétude des Modes TE et TM pour un Guide d'Ondes Optiques Planaire. Rapport de Recherche INRIA 385, INRIA, Paris, France, 1985.

[8] C. HAZARD AND M. LeNOIR, On the solution of time-harmonic scattering problems for Maxwell's equations, SIAM J. Math. Anal., 27 (1996), pp. 1597-1630.

[9] P. Joly AND C. PoIRIER, Mathematical analysis of electromagnetic open wave-guides, Math. Model. Numer. Anal., 29 (1995), pp. 505-575.

[10] D. Marcuse, Theory of Dielectric Optical Waveguides, Academic Press, New York, 1974.

[11] J. C. NÉdÉLEC, Approximation des Equations Intégrales en Mécanique et en Physique, Rapport interne de l'Ecole Polytechnique, Paris, France, 1977.

[12] C. Poirier, Guides d'Ondes Electromagnétiques Ouverts: Etude Mathématique et Numérique, Ph.D. thesis, Université de Nantes, Nantes, France, 1994.

[13] K. Ramdani, Lignes Supraconductrices: Analyse Mathématique et Numérique, Ph.D. thesis, Université de Paris VI, Paris, France, 1999.

[14] M. Reed And B. Simon, Methods of Modern Mathematical Physics. IV. Analysis of Operators, Academic Press, New York, 1978.

[15] P. Tixador, Les Supraconducteurs, Hermès, Paris, 1995.

[16] C. Weber, A local compactness theorem for Maxwell's equations, Math. Methods Appl. Sci., 2 (1967), pp. 12-25.

[17] R. WEDER, Absence of eigenvalues of the acoustic propagator in deformed wave guides, Rocky Mountain J. Math., 18 (1988), pp. 495-503. 